JOURNAL OF

SYMPLECTIC GEOMETRY

Volume 5, Number 2, 221-247, 2007

\title{
TEST CONFIGURATIONS FOR K-STABILITY AND GEODESIC RAYS
}

\author{
Duong H. Phong and Jacob Sturm
}

Let $X$ be a compact complex manifold, $L \rightarrow X$ an ample line bundle over $X$, and $\mathcal{H}$ the space of all positively curved metrics on $L$. We show that a pair $\left(h_{0}, T\right)$ consisting of a point $h_{0} \in \mathcal{H}$ and a test configuration $T=(\mathcal{L} \rightarrow \mathcal{X} \rightarrow \mathbf{C})$, canonically determines a weak geodesic ray $R\left(h_{0}, T\right)$ in $\mathcal{H}$ which emanates from $h_{0}$. Thus a test configuration behaves like a vector field on the space of Kähler potentials $\mathcal{H}$. We prove that $R$ is non-trivial if the $\mathbf{C}^{\times}$action on $X_{0}$, the central fiber of $\mathcal{X}$, is non-trivial. The ray $R$ is obtained as limit of smooth geodesic rays $R_{k} \subseteq \mathcal{H}_{k}$, where $\mathcal{H}_{k} \subseteq \mathcal{H}$ is the subspace of Bergman metrics.

\section{Dedicated to Dusa McDuff}

\section{Introduction}

Let $X$ be a compact complex manifold. According to a basic conjecture of Yau [33], the existence of canonical metrics on $X$ should be equivalent to a stability condition in the sense of geometric invariant theory. A version of this conjecture, due to Tian [31] and Donaldson [14], says that if $L \rightarrow X$ is an ample line bundle, then $X$ has a metric of constant scalar curvature in $c_{1}(L)$ if and only if the pair $(X, L)$ is K-stable, that is, if and only if the Futaki invariant $F(T)$ is negative for each non-trivial test configuration $T$. In particular, $F(T)<0$ for all such $T$ should imply that the K-energy $\nu: \mathcal{H} \rightarrow \mathbf{R}$ is bounded below, where $\mathcal{H}$ is the space of all positively curved metrics on $L$.

Now it is well known that the K-energy is convex along geodesics of $\mathcal{H}$ (Donaldson [12]). Thus, if $h_{0} \in \mathcal{H}$ and if $R:(-\infty, 0] \rightarrow \mathcal{H}$ is a smooth geodesic ray emanating from $h_{0}$, then the restriction of $\nu$ to $R$ is a smooth convex function $\nu_{R}:(-\infty, 0] \rightarrow \mathbf{R}$ and hence $\lim _{t \rightarrow-\infty} \dot{\nu}_{R}=a(R)$ is well defined (here $\dot{\nu}_{R}$ is the time derivative of the K-energy). In particular, if $a(R)<0$, then $\nu$ is bounded below on the ray $R$. 
We are thus led to the following plan for relating K-stability to lower bounds for the K-energy. Given a non-trivial test configuration $T=(\mathcal{L} \rightarrow$ $\mathcal{X} \rightarrow \mathbf{C})$ and a point $h_{0} \in \mathcal{H}$,

(A) Associate to $\left(h_{0}, T\right)$ a canonical non-trivial geodesic ray $R\left(T, h_{0}\right)$ emanating from $h_{0}$.

(B) Prove that the quantity $d(T)$ defined by $\lim _{t \rightarrow-\infty} \dot{\nu}_{R}=F(T)+d(T)$ satisfies $d(T)=0$ if $X_{0}$, the central fiber of $\mathcal{X}$, has no multiplicity, and that $F(T)<0$ implies $F(T)+d(T)<0$.

If this plan could be implemented, then $F(T)<0$ for a single test configuration $T$ would imply that $\nu$ is bounded below on the ray $R\left(T, h_{0}\right)$. And the K-stability of $(X, L)$ would imply that $\nu$ is bounded below on all the rays $R\left(T, h_{0}\right)$ emanating from $h_{0}$.

In this paper, we take a step in the direction of the plan outlined above. For step (A), we start with an arbitrary test configuration $T$ and an arbitrary point $h_{0} \in \mathcal{H}$. We associate to this data a weak geodesic $R\left(h_{0}, T\right)$ which is upper semi-continuous (but may not be smooth). If the $\mathbf{C}^{\times}$action on $X_{0}$ is non-trivial (in particular, if $F(T) \neq 0$ ), then we show that $R\left(h_{0}, T\right.$ ) is a non-trivial geodesic.

Our assignment of the weak geodesic $R\left(h_{0}, T\right)$ to each point $h_{0} \in \mathcal{H}$ is canonical. Thus a test configuration can be viewed as a (weak) vector field on $\mathcal{H}$. Even though the precise regularity properties of this vector field are not yet known, it is an intrinsic object which can be expected to play a role in future developments.

We also provide evidence for step (B). The ray $R\left(h_{0}, T\right)$ is constructed as a limit of Bergman geodesic rays $h(t ; k)$. Under certain geometric conditions, (which are necessary for our proofs, but we expect can be removed) we observe that the limit of the K-energy time derivative along $h(t ; k)=h_{0} e^{-\phi(t ; k)}$ converges to the Futaki invariant $F(T)$ as $k \rightarrow \infty$ if $X_{0}$ is multiplicity free.

After raising $\mathcal{L}$ and $L$ to sufficiently high powers, we may assume that $L$ is very ample, that $H^{0}(X, L)$ generates $\oplus_{k=0}^{\infty} H^{0}\left(X, L^{k}\right)$, and that $\mathcal{L}$ has exponent one (note that raising the power of the line bundle will just amount to a reparametrization of the geodesic). These assumptions will be made throughout this paper.

Our main results are Theorems 1 and 2 below, with relevant notation provided in $\S 4$.

Theorem 1.1. Let $L \rightarrow X$ be a very ample line bundle, $h_{0}$ a positively curved metric on $L$, and $T$ a test configuration for $(X, L)$. Let

$$
\phi_{t}=\lim _{k \rightarrow \infty}\left(\sup _{l \geq k}[\phi(t ; l)]\right)^{*}
$$


Then $h(t)=h_{0} e^{-\phi_{t}}$ is a weak geodesic ray emanating from $h_{0}$. Here we make use of the notation $u^{*}\left(\zeta_{0}\right)=\lim _{\epsilon \rightarrow 0} \sup _{\left|\zeta-\zeta_{0}\right|<\epsilon} u(\zeta)$ for any locally bounded $u: X \times(-\infty, 0] \rightarrow \mathbf{R}$.

Theorem 1.2. Assume that the action of $\mathbf{C}^{\times}$on $X_{0}$ is non-trivial. Then the weak geodesic defined by $\phi_{t}$ in Theorem 1.1 is non-trivial.

We note that the $\mathbf{C}^{\times}$action on $X_{0}$ is non-trivial if the Futaki invariant $F(T)$ of the test configuration $T$ does not vanish.

The following Theorem 1.3 is a direct consequence of the work of Tian [31] and Paul-Tian [22].

Theorem 1.3. Assume that the test configuration can be equivariantly imbedded in a proper family $\mathcal{X} \rightarrow B$, where $\mathcal{X}$ and $B$ are smooth compact manifolds with the property that the Chern class map $\operatorname{Pic}(B) \rightarrow H^{2}(B, \mathbf{Z})$ is injective and $X_{0}$ is multiplicity free. Then, for each $k>0$,

$$
\lim _{t \rightarrow-\infty} \dot{\nu}_{k}=F(T) \text {. }
$$

Here $\nu_{k}$ is the restriction of $\nu$ to the Bergman geodesic $h(t ; k)$.

Remark. Theorem 1.1 holds in a wider context than that stated above; our proofs show that one can associate a weak geodesic ray to an arbitrary traceless hermitian matrix $A \in \operatorname{gl}\left(H^{0}(X, L)\right)$ with rational eigenvalues. This can be reduced to the integer case by a base change $t \rightarrow t^{N}$ for some large integer $N$.

To define what is meant by a weak geodesic, we start by recalling that $\mathcal{H}$ is an infinite-dimensional symmetric space with respect to its natural Riemannian structure (see Mabuchi [19], Semmes [29], and Donaldson $[\mathbf{1 1}-\mathbf{1 3}, \mathbf{1 5}])$. Furthermore, the geodesic equation for $h_{0} e^{-\phi_{t}}$ is equivalent to the degenerate Monge-Ampère equation

$$
\Omega^{n+1}=0 \quad \text { on } X \times A,
$$

where $A \subseteq \mathbf{C}$ is an annulus (in the case of a geodesic segment) or a punctured disk (in the case of a geodesic ray). Here $\Omega=\Omega_{\phi}$ is the smooth (1,1)-form on $X \times A$ determined by: $\Omega=\Omega_{0}+\frac{\sqrt{-1}}{2} \partial \bar{\partial} \Phi$, where $\Omega_{0}=p_{1}^{*} \omega_{0}, \omega_{0}$ is the curvature of $h_{0}, p_{1}(x, w)=x, \Phi(x, w)=\phi_{t}(x)$, and $t=\log |w|$. A weak geodesic $\phi_{t}$ is one for which $\Omega_{\phi}$ is a plurisubharmonic, locally bounded, solution to (1.3) in the sense of pluripotential theory $[\mathbf{2}]$. (Note that the Monge-Ampère operator $\Omega^{n+1}$ is well defined for such potentials.) We expect the solution constructed in Theorem 1.1 to be of class $C^{1,1}$, but this has not yet been established at the present time.

The problem of constructing geodesic rays from test configurations has been considered previously by Arezzo-Tian [1]. They show that, if the central fiber of the test configuration $T$ is smooth, then one can use the 
Cauchy-Kowalevska theorem to find a local analytic solution near infinity to the geodesic equation, and in this way, they construct a geodesic ray $R(T)$ in $\mathcal{H}$. In fact, they construct a family of rays $R_{j}(T)$, where $j$ ranges over certain free parameters which determine the power series coefficients. These rays have the advantage of being real-analytic, but it does not appear that their origins can be prescribed by this method. Moreover, the relation of $R_{j}(T)$ to $F(T)$ is unclear.

We now provide an outline of the paper. The starting point is the approximation theorem for Kähler metrics by Bergman metrics. For $k \geq 1$, the space $\mathcal{H}_{k} \subseteq \mathcal{H}$ of Bergman metrics associated to $L^{k}$ is a finite-dimensional symmetric Riemannian sub-manifold. If $h \in \mathcal{H}$ and $h(k) \in \mathcal{H}_{k}$ is the associated Bergman metric, then the theorem of Tian-Yau-Zelditch $[\mathbf{3 1}, \mathbf{3 4}, \mathbf{3 5}]$ implies $h(k) \rightarrow h$ in the $C^{\infty}$ topology.

Now fix $h_{0}, h_{1} \in \mathcal{H}$, a pair of distinct elements, and let $h(t ; k)$ be the unique smooth geodesic segment in $\mathcal{H}_{k}$ defined by the conditions $h(0 ; k)=$ $h_{0}(k)$ and $h(1 ; k)=h_{1}(k)$. It was proved in $[\mathbf{2 7}]$ that the sequence $h(t ; k)$ converges uniformly, in the weak $C^{0}$ sense of Theorem 1.1, to a weak geodesic segment $h(t)$ in $\mathcal{H}$ with the property: $h(0)=h_{0}$ and $h(1)=h_{1}$. Moreover, $h(t)$ equals the $C^{1,1}$ geodesic joining $h_{0}$ to $h_{1}$, whose existence was established by Chen $[\mathbf{9}]$. We note that another approximation of the $C^{1,1}$ geodesic by potentials $\tilde{h}(t ; k)$ in $c_{1}(L)+\frac{1}{k} c_{1}\left(K_{X}\right)$ has been very recently constructed by Berndtsson $[\mathbf{4}, \mathbf{5}]$.

The proof of Theorem 1.1 follows the method of [27]. First, we construct a geodesic ray $h(t ; k)=h_{0} e^{-\phi(t ; k)}$ with $h(0 ; k)=h_{0}(k)$ that "points in the direction of $T$ ". Then we prove that

$$
\int_{X \times A} \Omega_{k}^{n+1}=O\left(k^{-1}\right)
$$

where $\Omega_{k}$ is associated to $\phi(t ; k)$. This step relies on the ideas developed in the recent work of Donaldson [16]. It also requires some estimates on test configurations, which include the following very simple, but basic estimate for the endomorphisms $A_{k}$ on $H^{0}\left(X_{0}, L_{0}^{k}\right)$ determined by a test configuration,

$$
\left\|A_{k}\right\|_{\text {op }}=O(k) \text {. }
$$

Next, we use the methods of pluripotential theory to establish the convergence of the $\phi(t ; k)$. In the case of geodesic rays, the annulus $A$ is actually a punctured disk, and the boundary behavior at the puncture has to be treated carefully, by controlling the asymptotics for the $\phi(t ; k)$ at the puncture.

For Theorem 1.2, we show that, when the test configuration is non-trivial, the sup norm of $\phi_{t}$ goes to $\infty$ near the puncture. This implies that the geodesic is non-trivial. A key ingredient is Donaldson's formula [16] for the leading coefficient of $\operatorname{Tr}\left(A_{k}^{2}\right)$. 
Regarding Theorem 1.3, we apply the formula in [31] which relates the metric of the CM line bundle $L_{\mathrm{CM}}$ to the K-energy. We then use [22] which relates the line bundle $\lambda_{\mathrm{CM}}$ on the Hilbert scheme to $L_{\mathrm{CM}}$.

We would like to add some references that have come to our attention since the posting of the first version of this paper. In a paper [23] which appeared shortly after ours, Paul and Tian present several results which include, in particular, Theorem 1.3. In fact, they actually prove a stronger result, in which the assumption on the injectivity of the Chern map is removed. As should be clear from its proof and as we already noted above, Theorem 1.3 was in any case an immediate consequence of their earlier work. In the recent paper [10], Chen shows that geodesic rays parallel to a given geodesic ray can be constructed under a certain assumption of tame ambient geometry. We would also like to note that constructions involving upper envelopes appear frequently in pluripotential theory, notably in the work of Kolodziej [17] (motivated, in part, by the work of Yau $[\mathbf{3 2}]$ ).

\section{Acknowledgement}

We would like to thank Julius Ross for some helpful conversations.

\section{Test configurations: preliminaries}

2.1. Definition. Let $L \rightarrow X$ be an ample line bundle over a compact complex manifold. A test configuration, as defined by Donaldson [14], consists of the following data:

(1) a scheme $\mathcal{X}$ with a $\mathbf{C}^{\times}$action $\rho$,

(2) a $\mathbf{C}^{\times}$equivariant line bundle $\mathcal{L} \rightarrow \mathcal{X}$ which is ample on all fibers,

(3) a flat $\mathbf{C}^{\times}$equivariant map $\pi: \mathcal{X} \rightarrow \mathbf{C}$ where $\mathbf{C}^{\times}$acts on $\mathbf{C}$ by multiplication,

satisfying the following. The fiber $X_{1}$ is isomorphic to $X$ and the pair $\left(X, L^{r}\right)$ is isomorphic to $\left(X_{1}, L_{1}\right)$ where, for $w \in \mathbf{C}, X_{w}=\pi^{-1}(w)$ and $L_{w}=\left.\mathcal{L}\right|_{X_{w}}$. After raising $\mathcal{L}$ and $L$ to sufficiently high powers, we may assume that $L$ is very ample, that $H^{0}(X, L)$ generates $\bigoplus_{k=0}^{\infty} H^{0}\left(X, L^{k}\right)$, and that $\mathcal{L}$ has exponent one. Thus we set $r=1$.

If $\tau \in \mathbf{C}^{\times}$and $w \in \mathbf{C}$, let $\rho_{k}(\tau, w): H^{0}\left(X_{w}, L_{w}^{k}\right) \rightarrow H^{0}\left(X_{\tau w}, L_{\tau w}^{k}\right)$ be the isomorphism induced by $\rho$. If $w=0$ we write $\rho_{k}(\tau, 0)=\rho_{k}(\tau)$. We also let $B_{k} \in \operatorname{End}\left(V_{k}\right)$ be defined by

$$
\rho_{k}\left(e^{t}\right)=e^{t B_{k}}
$$

for $t \in \mathbf{R}$, and $A_{k}$ the traceless part of $B_{k}$. The eigenvalues of $A_{k}$ are denoted by $\lambda_{0}^{(k)} \leq \lambda_{1}^{(k)} \leq \cdots \leq \lambda_{N_{k}}^{(k)}$, and the eigenvalues of $B_{k}$ are denoted by $\eta_{0}^{(k)} \leq \eta_{1}^{(k)} \leq \cdots \leq \eta_{N_{k}}^{(k)}$. Thus $\rho_{k}: \mathbf{C}^{\times} \rightarrow \mathrm{GL}\left(V_{k}\right)$, where $V_{k}=H^{0}\left(X_{0}, L_{0}^{k}\right)$. Let $d_{k}=\operatorname{dim} V_{k}$ and $w(k)=\operatorname{Tr}\left(B_{k}\right)$, the weight of the induced action on 
$\operatorname{det}\left(V_{k}\right)$. Then, as was observed in [14], there is an asymptotic expansion

$$
\frac{w(k)}{k d_{k}}=F_{0}+F_{1} k^{-1}+F_{2} k^{-2}+\cdots \quad \text { as } k \rightarrow \infty
$$

The Donaldson-Futaki invariant $F(T)$, or simply Futaki invariant, of $T$ is defined by the formula: $F(T)=F_{1}$.

2.2. Equivariant imbeddings of test configurations. The construction of the Bergman geodesics associated to a test configuration $T$ relies on the existence of an equivariant, unitary imbedding of $T$ into projective space, whose existence was first established by Donaldson [16]. In this section, we begin by recalling the statement of Donaldson's result.

Let $T$ be a test configuration of exponent $r=1$ for the pair $(X, L)$. For $k$ large, since $L$ is very ample, we have canonical compatible imbeddings $\iota_{k}: X_{1} \subseteq \mathbf{P}\left(H^{0}\left(X_{1}, L_{1}^{k}\right)^{*}\right)$ and $\iota_{k}: L_{1}^{k} \hookrightarrow O_{1}(1)$, where $O_{w}(1) \rightarrow$ $\mathbf{P}\left(H^{0}\left(X_{w}, L_{w}^{k}\right)^{*}\right)$ is the hyperplane line bundle, where $H^{0}\left(X_{w}, L_{w}^{k}\right)^{*}$ is the dual of $H^{0}\left(X_{w}, L_{w}^{k}\right)$.

One can show that the bundle $\pi_{*} \mathcal{L}^{k} \rightarrow \mathbf{C}$ has an equivariant trivialization and thus the test configuration has an equivariant imbedding into projective space. To be precise, let $\Theta$ be an arbitrary vector space isomorphism $\Theta: H^{0}\left(X_{0}, L_{0}^{k}\right) \rightarrow H^{0}\left(X_{1}, L_{1}^{k}\right)$, let $\mathcal{X}^{\times}=\pi^{-1}\left(\mathbf{C}^{\times}\right)$and let $\mathcal{L}^{\times}=\left.\mathcal{L}\right|_{\mathcal{X}} \times$ Define an imbedding $I_{\Theta}:\left(\mathcal{L}^{\times}\right)^{k} \hookrightarrow O_{0}(1) \times \mathbf{C}^{\times}$by the formula

$$
I_{\Theta}(\rho(\tau) l)=\left[\left(\rho_{k}(\tau) \Theta^{*}\left(\iota_{k}(l)\right), \tau\right],\right.
$$

where $\tau \in \mathbf{C}^{\times}, l \in L_{1}^{k}$ and $\Theta^{*}: O_{1}(1) \rightarrow O_{0}(1)$ is the isomorphism induced by the dual vector space isomorphism $\Theta^{*}: H^{0}\left(X_{1}, L_{1}^{k}\right)^{*} \rightarrow H^{1}\left(X_{0}, L_{0}^{k}\right)^{*}$. We similarly define the imbedding $I_{\Theta}: \mathcal{X}^{\times} \hookrightarrow \mathbf{P}\left(H^{0}\left(X_{0}, L_{0}^{k}\right)\right) \times \mathbf{C}^{\times}$. Then we say $\Theta: H^{0}\left(X_{0}, L_{0}^{k}\right) \rightarrow H^{0}\left(X_{1}, L_{1}^{k}\right)$ is a "regular generator of $T$ " if $I_{\Theta}$ extends to an imbedding $\mathcal{L}^{k} \hookrightarrow O_{0}(1) \times \mathbf{C}$ which restricts, over the central fiber, to the canonical embedding $L_{0}^{k} \hookrightarrow O_{0}(1)$.

Next let $h$ be a fixed metric on $L$. It is shown in [16] that there exists an regular generator $\Theta$ which respects $h$ structure in the following sense. The metric $h$ defines a hermitian metric $H_{k}$ on $H^{0}\left(X, L^{k}\right)$ by the formula $\left\langle s, s^{\prime}\right\rangle=\int_{X}\left(s, s^{\prime}\right)_{h^{k}} \omega^{n}$, where $\omega$ is the curvature of $h$. If $\Theta$ is a regular generator of $T$, then we can use the isomorphism $\Theta: V_{k} \rightarrow H^{0}\left(X, L^{k}\right)$ to define a metric on $V_{k}$, which we call $H_{k}(\Theta)$. Let $B_{k}$ be the endomophism of $V_{k}$ defined by: $\rho_{k}\left(e^{t}\right)=e^{t B_{k}}$ for $t \in \mathbf{R}$. We say $\Theta$ is a regular hermitian generator if $B_{k}$ is hermitian with respect to $H_{k}(\Theta)$. In other words, $\Theta$ is regular hermitian if $\rho_{k}(\tau): V_{k} \rightarrow V_{k}$ is an isometry for $|\tau|=1$.

In [16, Lemma 2], the following is proved.

Lemma 2.1. Let $T$ be a test configuration for $(X, L)$ and $h$ a positively curved metric on $L$. Then there exists $\Theta$, a regular hermitian generator for $T$. The metric $H_{k}=H_{k}(\Theta)$ is independent of the choice of such a $\Theta$. 
Moreover, the map $\Theta: V_{k} \rightarrow H^{0}\left(X_{1}, L_{1}^{k}\right)$ is unique up to an isometry of $V_{k}$ which commutes with $B_{k}$.

There are actually several closely related other versions of this lemma, namely Lemmas 2.3 and 2.7 below. See the remark after the statement of Lemma 2.3 for the precise relationship between these versions and the version appearing in $[\mathbf{1 6}]$.

For the sake of exposition and completeness, we shall provide a complete proof of Lemma 2.1 (which is of course essentially the one which appears in $[\mathbf{1 6}]$ ).

Let $E \rightarrow \mathbf{C}$ be an algebraic vector bundle of rank $r$. Then $E(\mathbf{C})$, the space of global sections of $E$, is a free $\mathbf{C}[t]$ module of rank $N+1$. A "trivialization of $E$ " is just a choice of ordered basis $S_{0}, \ldots, S_{N}$ of the $\mathbf{C}[t]$ module $E(\mathbf{C})$.

If $S_{0}, \ldots, S_{N}$ is a trivialization of $E$, and if $t \in \mathbf{C}$ then $S_{0}(t), \ldots, S_{N}(t)$ is a basis of the fiber $E_{t}$, and so we have a well defined isomorphism $\phi_{t_{2}, t_{1}}$ : $E_{t_{1}} \approx E_{t_{2}}$ for any pair $t_{1}, t_{2} \in \mathbf{C}$, which takes the basis $S_{j}\left(t_{1}\right)$ to the basis $S_{j}\left(t_{2}\right)$. The collection $\left\{\phi_{t_{2}, t_{1}}\right\}$ defines a regular cocycle, that is: $\phi_{t_{3}, t_{2}} \phi_{t_{2}, t_{1}}=$ $\phi_{t_{3}, t_{1}}$ and for every $e \in E_{t_{1}}$, the map $t \mapsto \phi_{t, t_{1}}(e)$ is a global section of $E$. Conversely, a regular cocycle $\phi_{t_{2}, t_{1}}$ defines a trivialization of $E$.

Now suppose $E \rightarrow \mathbf{C}$ is a vector bundle with a $\mathbf{C}^{\times}$action, covering the usual action of $\mathbf{C}^{\times}$on $\mathbf{C}$. This means that we are given an algebraic map $\rho: \mathbf{C}^{\times} \rightarrow \operatorname{Aut}(E \rightarrow \mathbf{C})$. Thus, if $\tau \in \mathbf{C}^{\times}$then $\rho(\tau): E \rightarrow E$ is a function with the following properties.

(1) The function $\rho(\tau)$ maps the fiber $E_{t}$ into the fiber $E_{\tau t}$, that is: $\pi(\rho(\tau) e)=\rho(\tau) \pi(e)$.

(2) The function $\rho(\tau): E_{t} \rightarrow E_{\tau t}$ is an isomorphism of vector spaces.

(3) If $\tau_{1}, \tau_{2} \in \mathbf{C}^{\times}$, then $\rho\left(\tau_{1} \tau_{2}\right)=\rho\left(\tau_{1}\right) \rho\left(\tau_{2}\right)$.

(4) The map $\mathbf{C}^{\times} \times E \rightarrow E$ given by $(\tau, e) \rightarrow \rho(\tau) e$ is algebraic.

Let $S_{0}, \ldots, S_{N}$ be a basis of global sections for $E$. If $S: \mathbf{C} \rightarrow E$ is an arbitrary global section, and if $\tau \in \mathbf{C}^{\times}$, then $S^{\rho(\tau)}(t)=\rho(\tau)^{-1} S(\tau t)$ is also a global section. Hence, there is a matrix $A(\tau, t) \in \operatorname{GL}\left(N+1, \mathbf{C}\left[\tau, \tau^{-1}, t\right]\right)$ with the property:

$$
\underline{S}^{\rho(\tau)}=A(\tau, t) \underline{S},
$$

where $\underline{S}$ is the column vector whose components are the $S_{j}$. Note that

$$
\begin{aligned}
\underline{S}^{\rho\left(\tau_{2} \tau_{1}\right)} & =\rho\left(\tau_{2} \tau_{1}\right)^{-1} \underline{S}\left(\tau_{2} \tau_{1} x\right)=\rho\left(\tau_{1}\right)^{-1} A\left(\tau_{2}, \tau_{1} t\right) \underline{S}\left(\tau_{1} x\right) \\
& =A\left(\tau_{2}, \tau_{1} t\right) A\left(\tau_{1}, t\right) \underline{S}(x)
\end{aligned}
$$

where, in the last equality, we are using the fact that $\rho\left(\tau_{1}\right)^{-1}$ is linear on the fibers. Hence:

$$
A\left(\tau_{2} \tau_{1}, t\right)=A\left(\tau_{2}, \tau_{1} t\right) A\left(\tau_{1}, t\right)
$$

In particular, if $A(\tau)=A(\tau, 0)$ then $A(\tau): \mathbf{C}^{\times} \rightarrow \mathrm{GL}(N+1, \mathbf{C})$ is a one parameter subgroup. 
With these preliminaries in place, we now show that if $E \rightarrow \mathbf{C}$ is an vector bundle with $\mathbf{C}^{\times}$action, then $E$ has a $\mathbf{C}^{\times}$equivariant trivialization.

Lemma 2.2. Let $E \rightarrow \mathbf{C}$ be a vector bundle of rank $r=N+1$ with a $\mathbf{C}^{\times}$action. Then there exists a basis of global sections $S_{0}, \ldots, S_{N}$ such that $A(\tau, t)$ is independent of $t$, that is, $A(\tau, t)=A(\tau, 0) \equiv A(\tau)$. In other words, there exists a regular cocycle $\left\{\phi_{t_{2}, t_{1}}\right\}$ satisfying

$$
\rho(\tau) \phi_{t_{2}, t_{1}} \rho(\tau)^{-1}=\phi_{\tau t_{2}, \tau t_{1}}
$$

The basis $S_{0}, \ldots, S_{N}$ is unique up to change of basis matrices $M(t) \in$ $\mathrm{GL}(N+1, \mathbf{C}[t])$ with the property: $M(\tau t)=A(\tau) M(t) A(\tau)^{-1}$.

Proof. Choose any $\mathbf{C}[t]$ basis $S_{0}, \ldots, S_{N} \in E(\mathbf{C})$ and define $A(\tau, t) \in$ $\mathrm{GL}\left(N+1, \mathbf{C}\left[\tau, \tau^{-1}, t\right]\right)$ as in equation (2.4). Thus $\operatorname{det}(A(\tau, t))=a \tau^{p}=$ $\operatorname{det}(A(\tau))$ for some integer $p$ and some $a \in \mathbf{C}^{\times}$. Now consider the set

$$
\mathcal{S}=\left\{S_{j}^{\rho(\tau)}: \tau \in \mathbf{C}^{\times}, 0 \leq j \leq N\right\}
$$

Let $V \subseteq E(\mathbf{C})$ be the complex vector space generated by $\mathcal{S}$. We claim that $V$ is finite-dimensional and invariant under the action of $\mathbf{C}^{\times}$. In fact, since $\underline{S}^{\rho(\tau)}=A(\tau, t) \underline{S}$, we see that the $S_{j}^{\rho(\tau)}$ are all linear combinations, with $\mathbf{C}$ coefficients, of elements in the set $\left\{t^{m} S_{j}: 0 \leq j \leq N, 0 \leq m \leq M\right\}$, where $M$ is chosen so that the entries of $A(\tau, t)$, which are polynomials in $t$ with coefficients in $A\left[\tau, \tau^{-1}\right]$, all have degree at most $M$.

Choose a basis $\left\{T_{\mu} ; 0 \leq \mu \leq K\right\}$ of $V$ with the property $T_{\mu}^{\rho(\tau)}=\tau^{l_{\mu}} T_{\mu}$ for some integers $l_{\mu}$. Choose $\mu_{j}, 0 \leq j \leq N$ such that $T_{\mu_{j}}(0)$ are linearly independent. This can certainly be done since the $T_{\mu}$ span $V$, and $V$ contains the $S_{j}$. Let $\underline{T}$ be the column vector consisting of the $T_{\mu_{j}}$. Then $\underline{T}(t)=$ $C(t) \underline{S}(t)$ for some $(N+1) \times(N+1)$ matrix $C(t)$ with coefficeints in $\mathbf{C}[t]$, for which $C(0)$ is invertible. The existence of such a matrix is guaranteed by the fact that the $S_{j}$ form a $\mathbf{C}[t]$ basis of $E(\mathbf{C})$. Replacing $\underline{S}$ by $C(0) \underline{S}$ does not change $V$ and allows us to assume $C(0)=I$. Now

$$
\underline{T}^{\rho(\tau)}(t)=\rho(\tau)^{-1} \underline{T}(\tau t)=\rho(\tau)^{-1} C(\tau t) \underline{S}(\tau t)=C(\tau t) A(\tau, t) \underline{S}(t) .
$$

On the other hand, $\underline{T}^{\rho(\tau)}(t)=U(\tau) \underline{T}(t)$, where $U(\tau)$ is diagonal with diagonal entries of the form $\tau^{l}$. Hence

$$
U(\tau) \underline{S}(0)=U(\tau) \underline{T}(0)=T^{\rho(\tau)}(0)=S^{\rho(\tau)}(0)=A(\tau) \underline{S}(0)
$$

so $U(\tau)=A(\tau)$. Thus

$$
C(\tau t) A(\tau, t) \underline{S}(t)=\underline{T}^{\rho(\tau)}(t)=A(\tau) \underline{T}(t)=A(\tau) C(t) \underline{S}(t)
$$

which implies: $A(\tau) C(t)=C(\tau t) A(\tau, t)$. Since $\operatorname{det}(A(\tau))=\operatorname{det}(A(\tau, t))$ for all $t$, we have $\operatorname{det}(C(\tau t))=\operatorname{det}(C(t))$ which means that $\operatorname{det}(C(t))$ is independent of $t$. Since $C(0)=I$, we conclude that $\operatorname{det}(C(t))=1$ and this implies that $\underline{T}$ is a $\mathbf{C}[t]$ basis of $E(\mathbf{C})$. This now establishes Lemma 2.2. 
At this point, we can prove the existence of a regular generator for $T$. Let $E=\pi_{*}\left(\mathcal{L}^{k}\right)^{*}$ so that $E_{t}=H\left(X_{t}, L_{t}^{k}\right)^{*}$. Then we define $\Theta^{*}: E_{1} \rightarrow E_{0}$ by the formula: $\Theta^{*}=\phi_{0,1}$ where $\phi_{t_{2}, t_{1}}$ satisfies (2.6), with $\rho(\tau)$ replaced by $\rho^{*}(\tau)=\rho\left(\tau^{-1}\right)^{*}$. One easily checks that $\Theta$ is a regular generator of $T$.

Lemma 2.3. Let $H_{1}$ be a hermitian metric on $E_{1}$. Then there is a unique equivariant trivialization $\phi_{t_{2}, t_{1}}$ such that $\rho(\tau)^{-1} \phi_{\tau, 1}: E_{1} \rightarrow E_{1}$ is an isometry for all $\tau \in \mathbf{C}^{\times}$with $|\tau|=1$.

Our formulation of Lemma 2.3 is somewhat different from that given in [16, Lemma 2]. The precise relation is as follows. Lemma 2 in [16] says that there is an equivariant trivialization $F: \mathbf{C} \times E_{0} \rightarrow E$ which takes a hermitian metric $H_{1}$ on the fiber at $\tau=1$ to a hermitian metric on the central fiber which is preserved by the action $S^{1} \subset \mathbf{C}^{\times}$on $E_{0}$. This is the content of Lemma 2.3 above, which is stated in terms of the cocycle $\phi_{t_{1}, t_{2}}$. The precise relationship is

$$
F(t, e)=\phi_{0, t}(e) .
$$

Proof of Lemma 2.3. Let $\left\{\phi_{t_{2}, t_{1}}\right\}$ be any equivariant trivialization. Consider the decomposition $E_{0}=\oplus V_{i}$ into eigenspaces for the action of $\mathbf{C}^{\times}$. Let $\tau^{w_{j}}$ be the restriction of $\rho(\tau)$ to the subspace $V_{j}$. We may assume that $w_{1}<$ $w_{2}<\cdots<w_{l}$. Thus $\sum_{j=1}^{l} w_{j} \operatorname{dim}\left(V_{j}\right)=N+1=\operatorname{dim}\left(E_{0}\right)$. Let $e_{0}, \ldots, e_{N}$ of $E_{0}$ be given by the union of the bases of the $V_{j}$ and define $S_{j}(t)=\phi_{t, 0}\left(e_{j}\right)$ and let $W_{i}=\phi_{t, 0}\left(V_{i}\right) \subseteq E_{1}$. Then $S_{0}, \ldots, S_{N}$ is a trivialization of $E \rightarrow \mathbf{C}$.

Let $A(\tau)$ be the diagonal matrix which represents the automorphism $\rho(\tau): E_{0} \rightarrow E_{0}$ with respect to the basis $e_{j}$. Then $A(\tau)$ also represents the automorphism $\rho(\tau)^{-1} \phi_{\tau, 1}: E_{1} \rightarrow E_{1}$ with respect to the basis $S_{j}(1)$. We want to modify the equivariant trivialization $\phi_{t_{2}, t_{1}}$ in such a way that this automorphism is an isometry. To do this, we must find a matrix $M(t) \in \mathrm{GL}(N+1, \mathbf{C}[t])$ satisfying:

(1) $M(\tau t) A(\tau) M(t)^{-1}=A(\tau)$ for all $t, \tau$;

(2) $M(1) S_{j}(1)$ is orthonormal with respect to $H$.

The first condition says that $M(t)$ is a block matrix with blocks $t^{w_{i}-w_{j}} \alpha_{i j}$, where $\alpha_{i j}$ is independent of $t$. Since $M(t) \in \mathrm{GL}(N+1, \mathbf{C}[t])$, this implies that $\alpha_{i j}=0$ if $i<j$. Thus $M(t)$ is lower block triangular. On the other hand, the usual Gram-Schmidt process allows us to choose an $M(1)$ of this form which satisfies condition (2). First choose an orthonormal basis of $W_{0}$. Then choose an orthonormal basis of $W_{0}^{\perp} \subseteq W_{0} \oplus W_{1}$, etc.

Finally we prove uniqueness. Let $M(t) \in \mathrm{GL}(N+1, \mathbf{C}[t])$ satisfy (1) and (2) and assume furthermore that the $e_{j}$ are orthonormal and that $M(0)=I$. Then we must show that $M(t)=I$ for all $t$. Since the $e_{j}$ are orthonormal, the matrix $M(1)$ is unitary. On the other hand, it is lower block triangular. This implies it is block diagonal. Since the $i, j$ block is of the form $t^{w_{i}-w_{j}} \alpha_{i j}$, 
and since $\alpha_{i j}=0$ for $i \neq j$, we see that $M(t)$ is independent of $t$ so $M(t)=$ $M(0)=I$. The lemma is proved.

Note that if $\phi$ is any equivariant trivialization, then $\rho(\tau)^{-1} \phi_{\tau, 1}: \mathbf{C}^{\times} \rightarrow$ $\mathrm{GL}\left(E_{1}\right)$ is a homomorphism:

$$
\rho\left(\tau_{1}\right)^{-1} \phi_{\tau_{1}, 1} \rho\left(\tau_{2}\right)^{-1} \phi_{\tau_{2}, 1}=\rho\left(\tau_{1} \tau_{2}\right)^{-1} \phi_{\tau_{1} \tau_{2}, \tau_{2}} \phi_{\tau_{2}, 1}=\rho\left(\tau_{1} \tau_{2}\right)^{-1} \phi_{\tau_{1} \tau_{2}, 1}
$$

where the first equality makes use of the equivariance property of $\phi$, and the second follows from the cocycle property of $\phi$. Thus the theorem can be restated as follows. There exists an equivariant trivialization $\phi$ such that $\rho(\tau)^{-1} \phi_{\tau, 1}: S^{1} \rightarrow \mathrm{GL}\left(E_{1}\right)$ is a unitary representation.

To deduce Lemma 2.1 from Lemma 2.3, we again define $\Theta^{*}=\phi_{0,1}$. Let $\tau \in \mathbf{C}^{\times}$be of unit length. Then to show $\rho_{k}(\tau)^{*}: V_{k}^{*} \rightarrow V_{k}^{*}$ is an isometry is equivalent, by definition of the metric on $V_{k}$, to showing $\left(\Theta^{*}\right)^{-1} \rho_{k}(\tau)^{*} \Theta^{*}: H\left(X_{1}, L^{k}\right)^{*} \rightarrow H\left(X_{1}, L^{k}\right)^{*}$ is an isometry. Thus we must show $\phi_{1,0} \rho_{k}(\tau)^{*} \phi_{0,1}=\phi_{1,0} \rho_{k}^{*}\left(\tau^{-1}\right) \phi_{0,1}$ is an isometry. But (2.6) implies

$$
\phi_{1,0} \rho_{k}^{*}\left(\tau^{-1}\right) \phi_{0,1}=\rho^{*}(\tau)^{-1} \phi_{\tau, 0} \phi_{0,1}=\rho^{*}(\tau)^{-1} \phi_{\tau, 1}
$$

which is an isometry by the result of Lemma 2.3. This proves Lemma 2.1.

\section{Estimates for test configurations}

3.1. Bounds for $A_{k}$. Let $T$ be a test configuration, and define the endomorphisms $A_{k}$ and $B_{k}$ and their eigenvalues $\lambda_{\alpha}^{(k)}$ and $\eta_{\alpha}^{(k)}$ as in $\S 2.1$. The following simple estimate for the operator norm $\left\|A_{k}\right\|_{\text {op }}$ of the endomorphisms $A_{k}$ plays an important role in the subsequent bounds for the total masses of the Monge-Ampère currents.

Lemma 3.1. There is a constant $C>0$ which is independent of $k$ such that $\left|\lambda_{\alpha}^{(k)}\right| \leq C k$ for all $k>0$ and all $\alpha$ such that $0 \leq \alpha \leq N_{k}$.

Proof. After applying Lemma 2.1 with $k=1$, we may assume that $\mathcal{X} \subseteq$ $\mathbf{P}^{m} \times \mathbf{C}, m=N_{1}+1$, and that $\rho(\tau)$ is a diagonal matrix in $\operatorname{GL}(m+1)$ whose entries are $\tau^{\eta_{0}}, \ldots, \tau^{\eta_{m}}$ where $\eta_{0} \leq \cdots \leq \eta_{m}$ are integers. The scheme $X_{0} \subseteq \mathbf{P}^{m}$ is defined by a homogenous ideal $I \subseteq \mathbf{C}\left[X_{0}, \ldots, X_{m}\right]$ and we write

$$
\mathbf{C}\left[X_{0}, \ldots, X_{m}\right] / I=\bigoplus_{k \geq 0} S_{k} / I_{k}
$$

where $S_{k} \subseteq \mathbf{C}\left[X_{0}, \ldots, X_{m}\right]$ is the space of polnomials which are homogeneous of degree $k$ and $I_{k}=S_{k} \cap I$. Then, for $k \gg 0$, we have $H^{0}\left(X_{0}, L_{0}^{k}\right)=$ $S_{k} / I_{k}$. The matrix $\rho(\tau)$ defines an automorphism of $\mathbf{C}\left[X_{0}, \ldots, X_{m}\right]$, determined by the formula: $X_{j} \mapsto \tau^{\eta_{j}} X_{j}$. This automorphism leaves $S_{k}$ and $I_{k}$ invariant, and thus it induces an automorphism of $S_{k} / I_{k}$ which is, by definition, the map $\rho_{k}(\tau)$.

The monomials of degree $k$ form a basis of $S_{k}$ which are eigenfunctions of $\rho(\tau)$. More precisely, if $X^{p}$ is a monomial, with $p=\left(p_{0}, \ldots, p_{m}\right)$ and 
$p_{0}+\cdots+p_{m}=k$, then we have $\rho(\tau) \cdot X^{p}=\tau^{p \cdot \eta} X^{\alpha}$. Since the monomials of degree $k$ span $S_{k} / I_{k}$, some subset form a basis of eigenvectors for that space. Thus the eigenvalues of the $B_{k}$ form a subset of $\left\{p \cdot \eta: p_{0}+\cdots+p_{m}=k\right\}$. On the other hand, for such an $p$, we clearly have $|p \cdot \eta| \leq \sup \left|\eta_{j}\right| \cdot k$ and this proves that

$$
\left|\eta_{\alpha}^{(k)}\right| \leq C k
$$

with $C=\sup _{0 \leq j \leq m}\left|\eta_{j}\right|$. On the other hand,

$$
\lambda_{\alpha}^{(k)}=\eta_{\alpha}^{(k)}-\frac{\operatorname{Tr}\left(B_{k}\right)}{N_{k}+1}=\eta_{\alpha}^{(k)}+O(k) .
$$

This proves Lemma 3.1.

\subsection{An alternative characterization of the Futaki invariant.}

3.2.1. The $F_{\omega}^{0}$ functional. Let $X$ be a compact complex manifold of dimension $n$ and $\omega=\omega_{0}$ a Kähler metric on $X$. Let $\mathcal{H}=\mathcal{H}_{\omega}$ be the space of Kähler potentials:

$$
\mathcal{H}_{\omega}=\left\{\phi \in C^{\infty}(X): \omega_{\phi}=\omega+\frac{\sqrt{-1}}{2} \partial \bar{\partial} \phi>0\right\} .
$$

The functionals $F_{\omega}^{0}, \nu_{\omega}: \mathcal{H} \rightarrow \mathbf{R}$ play an important role in Kähler geometry and are defined as follows:

$$
\begin{aligned}
F_{\omega}^{0}(\phi) & =-\frac{1}{n+1}\left(\int_{X} \omega^{n}\right)^{-1} \sum_{j=0}^{n} \int_{X} \phi \omega_{\phi}^{j} \omega^{n-j}, \\
& =-\frac{1}{n+1}\left(\int_{X} \omega^{n}\right)^{-1} E_{\omega}(\phi), \\
\nu_{\omega}(\phi) & =-\left(\int_{X} \omega^{n}\right)^{-1} \int_{0}^{1} \int_{X} \dot{\phi}(s-\hat{s}) \omega_{t}^{n} d t .
\end{aligned}
$$

Here $\phi_{t}, 0 \leq t \leq 1$, is a smooth path in $\mathcal{H}_{\omega}$ joining the potential $\phi_{0}$ for $\omega_{0}$ to $\phi=\phi_{1}$. Then a simple calculation shows

$$
\dot{E}_{\omega}\left(\phi_{t}\right)=(n+1) \int_{X} \dot{\phi}_{t} \omega_{\phi_{t}}^{n} \quad \text { and } \quad \ddot{E}_{\omega}\left(\phi_{t}\right)=(n+1) \int_{X}\left(\ddot{\phi}_{t}-\left|\partial \dot{\phi}_{t}\right|^{2}\right) \omega_{\phi_{t}}^{n} \text {. }
$$

Thus $E$ satisfies the cocycle property: $E_{\omega}(\phi)+E_{\omega_{\phi}}(\psi)=E_{\omega}(\phi+\psi)$. Note as well that if $f: Y \rightarrow X$ is a biholomorphic map, then

$$
E_{f^{*} \omega}(\phi \circ f)=E_{\omega}(\phi)
$$


3.2.2. The Chow weight and the Futaki invariant. Let $V$ be a finitedimensional vector space, $Z \subseteq \mathbf{P}(V)$ a smooth subvariety, and $B \in \operatorname{gl}(V)$. Then we wish to define the generalized Chow weight $\mu(Z, B) \in \mathbf{R}$. We start by assuming the $V=\mathbf{C}^{N+1}$ so that $B$ is a $(N+1) \times(N+1)$ matrix. Let $\omega_{\mathrm{FS}}$ be the Fubini-Study metric on $\mathbf{P}^{N}$. We shall also denote by $\omega_{\mathrm{FS}}$ the restriction of the Fubini-Study metric to $Z$. For $t \in \mathbf{R}$, let $\sigma_{t} \in \mathrm{GL}(N+1, \mathbf{C})$ be the matrix $\sigma_{t}=e^{t B}$ and let $\psi_{t}: \mathbf{P}^{N} \rightarrow \mathbf{R}$ be the function

$$
\psi_{t}(z)=\log \frac{\left|\sigma_{t} z\right|^{2}}{|z|^{2}} \text {. }
$$

Here we view $z$ as an element in $\mathbf{P}^{N}$ and, when there is no fear of confusion, a column vector in $\mathbf{C}^{N+1}$.

Then $\psi_{t}$ is a smooth path in $\mathcal{H}$. In fact, $\sigma_{t}^{*} \omega_{\mathrm{FS}}=\omega_{\mathrm{FS}}+\frac{\sqrt{-1}}{2} \partial \bar{\partial} \psi_{t}$. Define

$$
\mu(Z, B)=-\lim _{t \rightarrow-\infty} \dot{E}_{\omega_{\mathrm{FS}}}\left(\psi_{t}\right)=-\dot{E}(-\infty) .
$$

Note that the function $E(t)=E_{\omega_{\mathrm{FS}}}\left(\psi_{t}\right): \mathbf{R} \rightarrow \mathbf{R}$ is convex (see $[\mathbf{2 4}, \mathbf{2 5}]$ ), so the limit in (3.8) exists.

Next we compute the derivative of $E(t)$ :

$$
\begin{aligned}
\frac{d}{d t} E_{\omega_{\mathrm{FS}}}\left(\psi_{t}\right) & =(n+1) \int_{Z} \frac{z^{*} \sigma_{t}^{*} \cdot\left(B+B^{*}\right) \cdot \sigma_{t} z}{z^{*} \sigma_{t}^{*} \sigma_{t} z} \sigma_{t}^{*} \omega_{\mathrm{FS}}^{n} \\
& =(n+1) \int_{\sigma_{t}(Z)} \frac{z^{*} \cdot\left(B+B^{*}\right) \cdot z}{z^{*} z} \omega_{\mathrm{FS}}^{n}
\end{aligned}
$$

where, for $C$ a matrix with complex entries, we write $C^{*}={ }^{t} \bar{C}$. In particular,

$$
\left.\dot{E}_{\omega_{\mathrm{FS}}}\left(\psi_{t}\right)\right|_{t=0}=\dot{E}(0)=(n+1) \operatorname{Tr}\left(\left(B+B^{*}\right) \cdot M\right)
$$

where

$$
M_{\alpha \beta}=M_{\alpha \beta}(Z)=\int_{Z} \frac{z_{\alpha} \bar{z}_{\beta}}{\|z\|^{2}} \omega_{\mathrm{FS}}^{n} .
$$

Lemma 3.2. Let $V$ be a finite-dimensional complex vector space, $B \in \operatorname{gl}(V)$ and $Z \subseteq \mathbf{P}(V)$ a smooth subvariety. Let $\theta: V \rightarrow \mathbf{C}^{N+1}$ be an isomorphism. Then $\mu\left(\theta(Z), \theta B \theta^{-1}\right)$ is independent of $\theta$.

Proof. We make use of the formula of Zhang [36] and Paul [21] (see also $[\mathbf{2 4}, \mathbf{2 6}])$ : If $Z \subseteq \mathbf{P}^{N}(\mathbf{C})$ is a subvariety of dimension $n$ and degree $d$, let Chow $(Z) \in \mathbf{P}\left(H^{0}\left(\operatorname{Gr}\left(N-n, \mathbf{C}^{N+1}, O(d)\right)\right)\right)$ be the Chow point of $Z \subseteq \mathbf{P}^{N}$. If $B \in \operatorname{gl}(N+1, \mathbf{C}), \sigma_{t}=e^{t B}$, and $\psi_{\sigma_{t}}=\log \frac{\left|\sigma_{t}(z)\right|^{2}}{|z|^{2}}$ then

$$
E_{\omega_{\mathrm{FS}} \mid z}\left(\psi_{\sigma_{t}}\right)=\log \frac{\left\|\sigma_{t} \cdot \operatorname{Chow}(Z)\right\|^{2}}{\|\operatorname{Chow}(Z)\|^{2}}=\log \frac{\left\|\operatorname{Chow}\left(\sigma_{t} Z\right)\right\|^{2}}{\|\operatorname{Chow}(Z)\|^{2}}
$$

where $\|\cdot\|$ is the Chow norm defined on $H^{0}\left(\operatorname{Gr}\left(N-n, \mathbf{C}^{N+1}, O(d)\right)\right)$. 
Suppose $M \in \operatorname{GL}(N+1, \mathbf{C})$. Then

$$
E_{\left.\omega_{\mathrm{FS}}\right|_{M Z}}\left(\psi_{M \sigma_{t} M^{-1}}\right)=\log \frac{\left\|M \sigma_{t} M^{-1} \cdot \operatorname{Chow}(M Z)\right\|^{2}}{\|\operatorname{Chow}(M Z)\|^{2}} .
$$

Subtracting (3.12) from (3.13) we get

$$
\begin{aligned}
& E_{\left.\omega_{\mathrm{FS}}\right|_{M Z}}\left(\psi_{M \sigma_{t} M-1}\right)-E_{\omega_{\mathrm{FS}} \mid Z}\left(\psi_{\sigma_{t}}\right) \\
& \quad=\log \frac{\left\|M \sigma_{t} \cdot \operatorname{Chow}(Z)\right\|^{2}}{\left\|\sigma_{t} \cdot \operatorname{Chow}(Z)\right\|^{2}}-\log \frac{\|M \cdot \operatorname{Chow}(Z)\|^{2}}{\|\operatorname{Chow}(Z)\|^{2}}
\end{aligned}
$$

which is a bounded function of $t$, and hence the limit of its first derivative is zero. This proves Lemma 3.2.

Now let $Z \subseteq \mathbf{P}(V)$ and $B \in \operatorname{gl}(V)$. Let $\theta: V \rightarrow \mathbf{C}^{N+1}$ be an isomorphism and define $\mu(Z, B)=\mu\left(\theta(Z), \theta B \theta^{-1}\right)$. The lemma guarantees that this definition is unambiguous. Note that (3.12) shows that $\mu(Z, B)$ is just the usual Chow weight. (The Chow weight is normally defined only when $B$ is a traceless diagonalizable matrix with integer eigenvalues, but we find it convenient to work with this somewhat more general notion.)

If $\tau \in \mathrm{GL}(V)$ then

$$
\mu(\tau(Z), B)=\mu\left(\theta \tau(Z), \theta B \theta^{-1}\right)=\mu\left((\theta \tau)(Z),(\theta \tau) \tau^{-1} B \tau(\theta \tau)^{-1}\right) .
$$

We conclude that

$$
\mu(\tau(Z), B)=\mu\left(Z, \tau^{-1} B \tau\right) .
$$

In particular, if $\tau$ commutes with $B$, then $\mu(Z, B)=\mu(\tau(Z), B)$.

If we replace the functional $E$ by $\nu$, the $K$ energy functional, we may define a corresponding invariant $\tilde{\mu}(Z, B)$ for $Z \subseteq \mathbf{P}^{N}(\mathbf{C})$ and $B \in \mathrm{gl}$ $(N+1, \mathbf{C})$ :

$$
\tilde{\mu}(Z, B)=\lim _{t \rightarrow-\infty} \dot{\nu}_{\omega_{\mathrm{FS}}}\left(\psi_{t}\right) .
$$

It will be convenient for us to introduce an alternative characterization of the Futaki invariant. Fix, once and for all, an isomorphism $\kappa:\left(X, L^{r}\right) \rightarrow$ $\left(X_{1}, L_{1}\right)$. We continue to assume that $r=1$ (the case $r>1$ can be treated in a similar fashion). Then we have an induced isomorphism $H^{0}\left(X, L^{k}\right)=$ $H^{0}\left(X_{1}, L_{1}^{k}\right)$.

Let $\Theta$ be an equivariant trivialization of $\pi_{*} \mathcal{L}^{k}$. Then

$$
\left.I_{\Theta}\right|_{X_{1}}: X_{1} \hookrightarrow \mathbf{P}\left(H^{0}\left(X_{0}, L_{0}^{k}\right)\right) .
$$

Let $Z_{k} \subseteq \mathbf{P}\left(H^{0}\left(X_{0}, L_{0}^{k}\right)^{*}\right)$ be the image of $\left.I_{\Theta}\right|_{X_{1}}$ and $Z_{k}^{(0)}$ the image of the canonical imbedding $X_{0} \subseteq \mathbf{P}\left(H^{0}\left(X_{0}, L_{0}^{k}\right)^{*}\right)$. Note that $Z_{k}$ depends on the choice of $\Theta$, but that if $\Theta^{\prime}$ is another choice, then $\Theta^{\prime}=U \Theta$ where $U A_{k}=A_{k} U$, and thus the value $\mu\left(Z_{k}, A_{k}\right)$ is independent of the choice of equivariant $\Theta$. 
Lemma 3.3. We have

$$
F(T)=-c(X, \omega) \cdot \lim _{k \rightarrow \infty} \frac{\mu\left(Z_{k}, A_{k}\right)}{k^{n}},
$$

where $c(X, \omega)=\frac{1}{n !(n+1) !} \int_{X} \omega^{n}$.

Proof. Since this argument is implicit in Donaldson [14], we only briefly sketch the proof (see as well Ross-Thomas [28]). If $Z \subseteq \mathbf{P}^{N}$ and $\lambda: \mathbf{C}^{\times} \rightarrow$ $\mathrm{SL}(N+1, \mathbf{C})$ is a one parameter subgroup, let $A \in \operatorname{sl}(N+1)$ be such that $\lambda\left(e^{t}\right)=e^{t A}$ and $Z^{(0)}=\lim _{\tau \rightarrow 0} \lambda(\tau)(Z)$ (the flat limit) so $Z^{(0)} \subseteq \mathbf{P}^{N}$ is a subscheme of $\mathbf{P}^{N}$ with the same Hilbert polynomial as $Z$. Let $M_{0}=$ $\left.O(1)\right|_{Z^{(0)}}$. Then $\lambda(\tau)$ defines an automorphism of $H^{0}\left(Z^{(0)}, M_{0}^{p}\right)$ and we let $\tilde{w}(Z, A, p)$ be the weight of this action on $\operatorname{det}\left(H^{0}\left(X_{0}, M_{0}^{p}\right)\right)$. It is known that $\tilde{w}(p)$ is a polynomial in $p$ for $p$ large such that

$$
\tilde{w}(Z, A, p)=\frac{\mu(Z, A)}{(n+1) !} \cdot p^{n+1}+O\left(p^{n}\right) \quad \text { and } \quad \tilde{w}\left(Z^{(0)}, 1\right)=0
$$

(see, for example, Mumford [20]). Now let $T$ be a test configuration, let $r>0$ and consider $Z_{r} \subseteq \mathbf{P}\left(H^{0}\left(X_{0}, L_{0}^{r}\right)^{*}\right)$. Applying (3.17) to $Z=Z_{r}$, $A=r N_{r} A_{r}$ and $M_{0}=L_{0}^{r}$, we get

$$
\tilde{w}\left(Z_{r}, r N_{r} A_{r}, p\right)=\frac{\mu\left(Z_{r}, r N_{r} A_{r}\right)}{(n+1) !} \cdot p^{n+1}+O\left(p^{n}\right)
$$

On the other hand, since $M_{0}^{p}=L_{0}^{r p}$, we get, with $k=r p$ :

$$
\tilde{w}\left(Z_{r}, r N_{r} A_{r}, p\right)=w(k) r N_{r}-w(r) k N_{k}=e_{T}(r) k^{n+1}+O\left(k^{n}\right),
$$

where $e_{T}$ is a polynomial in $r$ of degree at most $n$. If follows from the definition of $F(T)$ that $-F(T)$ is the leading coefficient of $e_{T}(r)$. Comparing with (3.18) we get

$$
\lim _{r \rightarrow \infty} \frac{\mu\left(Z_{r}, r N_{r} A_{r}\right)}{r^{n} r^{n+1}(n+1) !}=-F(T)
$$

Since $r^{-n} N_{r}=\frac{1}{n !} \int \omega^{n}+O\left(r^{-1}\right)$, Lemma 3.3 follows.

\section{Completion of the proof of Theorem 1.2}

4.1. The Tian-Yau-Zelditch expansion. Let $L \rightarrow X$ be an ample line bundle over a compact complex manifold $X$. If $h$ is a smooth hermitian metric on $L$ then the curvature of $h$ is given by $\omega=R(h)=-\frac{\sqrt{-1}}{2} \partial \bar{\partial} \log h$. Let $\mathcal{H}$ be the space of positively curved hermitian metrics on $L$. Then $\mathcal{H}$ contains a canonical family of finite-dimensional negatively curved symmetric spaces $\mathcal{H}_{k}$, the space of Bergman metrics, which are defined as follows. For $k \gg 0$ and for $\underline{s}=\left(s_{0}, \ldots, s_{N_{k}}\right)$ an ordered basis of $H^{0}\left(X, L^{k}\right)$, let

$$
\iota_{\underline{s}}: X \hookrightarrow \mathbf{P}^{N_{k}}
$$


be the Kodaira imbedding given by $x \mapsto\left(s_{0}(x), \ldots, s_{N_{k}}(x)\right)$. Then we have a canonical isomorphism $\iota_{\underline{s}}: L^{k} \rightarrow \iota_{\underline{s}}^{*} O(1)$ given by

$$
\iota_{\underline{s}}(l)=\left[\left(\frac{s_{0}}{s}, \frac{s_{1}}{s}, \ldots, \frac{s_{N}}{s}\right) \mapsto \frac{l}{s}\right],
$$

where $l \in L^{k}$ and $s$ is any locally trivializing section of $L^{k}$.

Fix $h_{0} \in \mathcal{H}$. Let $h_{\mathrm{FS}}$ be the Fubini-study metric on $O(1) \rightarrow \mathbf{P}^{N_{k}}$ and let

$$
h_{\underline{s}}=\left(\iota_{\underline{s}}^{*} h_{\mathrm{FS}}\right)^{1 / k}=\frac{h_{0}}{\left(\sum_{\alpha=0}^{N_{k}}\left|s_{\alpha}\right|_{h_{0}^{k}}^{2}\right)^{1 / k}} .
$$

Note that the right-hand side of (4.2) is independent of the choice of $h_{0} \in \mathcal{H}$. In particular,

$$
\sum_{\alpha=0}^{N_{k}}\left|s_{\alpha}\right|_{h_{\underline{s}}^{k}}^{2}=1
$$

Let

$$
\mathcal{H}_{k}=\left\{h_{\underline{s}}: \underline{s} \text { a basis of } H^{0}\left(X, L^{k}\right)\right\} \subseteq \mathcal{H} .
$$

Then $\mathcal{H}_{k}=G L\left(N_{k}+1\right) / U\left(N_{k}+1\right)$ is a finite-dimensional negatively curved symmetric space sitting inside of $\mathcal{H}$. It is well known that the $\mathcal{H}_{k}$ are topologically dense in $\mathcal{H}$. If $h \in \mathcal{H}$ then there exists $h(k) \in \mathcal{H}_{k}$ such that $h(k) \rightarrow h$ in the $C^{\infty}$ topology. This follows from the Tian-Yau-Zelditch theorem on the density of states (Tian [30], Yau [33], and Zelditch [35]; see also Catlin [7] for corresponding results for the Bergman kernel). In fact, if $h \in \mathcal{H}$, then there is a canonical choice of the approximating sequence $h(k)$. Let $\underline{s}$ be a basis of $H^{0}\left(X, L^{k}\right)$ which is orthonormal with respect to the metrics $h$. In other words,

$$
\left\langle s_{\alpha}, s_{\beta}\right\rangle_{h}=\int_{X}\left(s_{\alpha}, s_{\beta}\right)_{h^{k}} \omega^{n}=\delta_{\alpha \beta} \quad \text { where } \omega=R(h) .
$$

The basis $\underline{s}$ is unique up to an element of $U\left(N_{k}+1\right)$. Define $\rho_{k}(h)=$ $\rho_{k}(\omega)=\sum_{\alpha}\left|s_{\alpha}\right|_{h^{k}}^{2}$. Then [35, Theorem 1], which is the $C^{\infty}$ version of the $C^{2}$ approximation result first estsablished in [30], says that for $h$ fixed, we have a $C^{\infty}$ asymptotic expansion as $k \rightarrow \infty$ :

$$
\rho_{k}(\omega) \sim k^{n}+A_{1}(\omega) k^{n-1}+A_{2}(\omega) k^{n-1}+\cdots .
$$

Here the $A_{j}(\omega)$ are smooth functions on $X$ defined locally by $\omega$ which can be computed in terms of the curvature of $\omega$ by the work of $\mathrm{Lu}[\mathbf{1 8}]$. In particular, it is shown there that

$$
A_{1}(\omega)=\frac{s(\omega)}{2 \pi},
$$

where $s(\omega)$ is the scalar curvature of $\omega$. 
Let $\underline{\hat{s}}=k^{-n / 2} \underline{s}$ and $h(k)=h_{\underline{\hat{s}}}$. Then (4.2) and (4.5) imply that

$$
\begin{aligned}
& \frac{h(k)}{h}=1-\frac{s(\omega)}{2 \pi} \cdot \frac{1}{k^{2}}+O\left(\frac{1}{k^{3}}\right), \quad \omega(k)=\omega+O\left(\frac{1}{k^{2}}\right), \\
& \phi(k)=\phi+O\left(\frac{1}{k^{2}}\right) .
\end{aligned}
$$

Here, as before, $\omega=R(h), \omega(k)=R(h(k)), h=h_{0} e^{-\phi}$, and $h(k)=h_{0} e^{-\phi(k)}$. In particular, $\omega_{0}+\frac{\sqrt{-1}}{2} \partial \bar{\partial} \phi(k)=\omega(k)=\frac{1}{k} \iota_{\underline{s}}^{*} \omega_{\mathrm{FS}}$.

Lemma 2.1 can now be conveniently reformulated as follows.

Lemma 4.1. Let $\rho: \mathbf{C}^{\times} \rightarrow \operatorname{Aut}(\mathcal{L} \rightarrow \mathcal{X} \rightarrow \mathbf{C})$ be a test configuration $T$ of exponent one for the pair $(X, L)$, where $L \rightarrow X$ is ample. Let $h_{0}$ be a positively curved metric on $L \rightarrow X$. Let $k$ be an integer such that $L^{k}$ is very ample. Then there is

(1) an orthonormal basis $\underline{s}=\left(s_{0}, \ldots, s_{N_{k}}\right)$ of $H^{0}\left(X, L^{k}\right)=H^{0}\left(X_{1}, L_{1}^{k}\right)$,

(2) an imbedding $I_{\underline{s}}:\left(\mathcal{L}^{k} \rightarrow \mathcal{X} \rightarrow \mathbf{C}\right) \hookrightarrow\left(O(1) \times \mathbf{C} \rightarrow \mathbf{P}^{N_{k}} \times \mathbf{C} \rightarrow \mathbf{C}\right)$, satisfying the following property: the imbedding $I_{\underline{s}}$ restricts to $\iota_{\underline{s}}$ on the fiber $L_{1}^{k}$ and $I_{\underline{s}}$ intertwines $\rho(\tau)$ and $\tau^{B_{k}}$. More precisely, for every $\tau \in \mathbf{C}^{\times}$and every $l_{w} \in L_{w}^{k}$,

$$
I_{\underline{s}}\left(\rho(\tau) l_{w}\right)=\left(\tau^{B_{k}} \cdot I_{\underline{s}}\left(l_{w}\right), \tau w\right),
$$

where $\tau^{B_{k}}$ is a diagonal matrix whose eigenvalues are the eigenvalues of $\rho_{k}(\tau): V_{k} \rightarrow V_{k}$.

The matrix $B_{k}$ is uniquely determined, up to a permutation of the diagnonal entries, by $k$ and the test configuration T. Moreover, the basis $\underline{s}$ is uniquely determined by $h_{0}$ and $T$, up to an element of $U\left(N_{k}+1\right)$ which commutes with $B_{k}$. The image of $X_{1}$ is $Z_{k} \subseteq \mathbf{P}^{N_{k}}$.

This lemma can be illustrated by the following simple example:

Example. Let $\lambda_{0}, \ldots, \lambda_{N}$ be a sequence of integers, and, for $\tau \in \mathbf{C}^{\times}$, let $\sigma(\tau)$ be the diagonal matrix whose entries are $\tau^{\lambda_{0}}, \ldots, \tau^{\lambda_{N}}$. Then $\sigma(\tau)$ defines maps $\sigma(\tau): \mathbf{P}^{N} \rightarrow \mathbf{P}^{N}$ as well as $\sigma(\tau): O(1) \rightarrow O(1)$.

Let $X \subseteq \mathbf{P}^{N}$ be a smooth projective variety and assume that for all $\tau \in \mathbf{C}^{\times}$we have $\sigma(\tau)(X)=X$. Let $L=\left.O(1)\right|_{X}$ so that $\sigma(\tau): L \rightarrow L$ and let $h$ be a hermitian metric on $L$ which is invariant under the $S^{1}$ action: $\sigma(\tau)^{*} h=h$ for all $\tau$ with $|\tau|=1$.

Now define a test configuration $T$ as follows: $\mathcal{X}=X \times \mathbf{C}$ and $\mathcal{L}=\pi_{1}^{*} L$, where $\pi_{1}: \mathcal{X} \rightarrow \mathbf{C}$ and $\pi_{2}: \mathcal{X} \rightarrow X$ are the projection maps. Here we let $\rho(\tau): \mathcal{X} \rightarrow \mathcal{X}$ be the map $\rho(\tau)(x, t)=(\sigma(\tau) x, \tau t)$. We wish to spell out the basis $\underline{s}$ and the imbedding $I_{\underline{s}}$ from Lemma 4.1.

To do this we fix $k \gg 1$ and let $\sigma_{k}: \mathbf{C}^{\times} \rightarrow H^{0}\left(X, L^{k}\right)$ be the action on $H^{0}\left(X, L^{k}\right)$ induced by $\sigma$. To describe $\sigma_{k}$ concretely, we define 
an action of $\mathbf{C}^{\times}$on $\mathbf{C}\left[X_{0}, \ldots, X_{N}\right]$ by the formula: $F^{\tau}\left(X_{0}, \ldots, X_{N}\right)=$ $F\left(\tau^{\lambda_{0}} X_{0}, \ldots, \tau^{\lambda_{N}} X_{N}\right)$. We let $R_{k} \subseteq \mathbf{C}\left[X_{0}, \ldots, X_{N}\right]$ be the space of polynomials which are homogeneous of degree $k$ and let $Z_{k} \subseteq R_{k}$ be the subspace which vanishes on $X$. Then $R_{k}$ and $Z_{k}$ are invariant under the $\mathbf{C}^{\times}$action, and thus there is a well defined $\mathbf{C}^{\times}$action $\sigma_{k}$ on $H^{0}\left(X, L^{k}\right)=R_{k} / Z_{k}$.

Now we decompose the vector space $H^{0}\left(X, L^{k}\right)=U_{1} \oplus \cdots \oplus U_{r}$, where the $U_{j}$ are the eigenspaces of the matrix $\sigma_{k}(\tau)$. This means that there are distinct integers $a_{j}$ such that $\sigma_{k}(\tau)\left(v_{j}\right)=\tau^{a_{j}} v_{j}$ for all $v_{j} \in V_{j}$. Since $h$ is invariant, we have $\left\langle v_{j}, v_{l}\right\rangle=\tau^{a_{j}-a_{l}}\left\langle v_{j}, v_{l}\right\rangle$ whenever $|\tau|=1$. Thus $V_{j}$ and $V_{l}$ are orthogonal if $j \neq l$.

Fix an orthonormal basis $B_{j}$ of each $V_{j}$, and let $\underline{s}$ be the basis of $H^{0}\left(X, L^{k}\right)$ defined by $\underline{s}=\left(B_{1}, \ldots, B_{r}\right)$. Define $I_{\underline{s}}:\left(L^{k} \times \mathbf{C} \rightarrow X \times \mathbf{C} \rightarrow \mathbf{C}\right) \hookrightarrow$ $\left(O(1) \times \mathbf{C} \rightarrow \mathbf{P}^{N_{k}} \times \mathbf{C} \rightarrow \mathbf{C}\right)$ as follows: $I_{\underline{s}}(x, t)=\left(s_{0}(x), \ldots, s_{N}(x) ; t\right)$.

4.2. Growth bounds for the Bergman geodesic rays. We make precise the notation which appears in Theorem 1.1. Let $L \rightarrow X$ be an ample line bundle over a compact complex manifold, and $\mathcal{H}$ the space of positively curved metrics on $L$. Let $h_{0} \in \mathcal{H}$ and let $T$ be a test configuration for the pair $(X, L)$ of exponent $r$. We wish to associate to the pair $\left(h_{0}, T\right)$ an infinite geodesic ray in $\mathcal{H}$ whose initial point is $h_{0}$. After replacing $L$ by $L^{r}$ we may assume, without loss of generality, that $r=1$ and that $L$ is very ample.

Let $k$ be a large positive integer and choose $\underline{s}$, an orthonormal basis of $H^{0}\left(X, L^{k}\right)$ as in Lemma 4.1. Define $A_{k}$ to be the traceless part of $B_{k}$ and let $\lambda_{0}^{(k)} \leq \lambda_{1}^{(k)} \leq \cdots \leq \lambda_{N_{k}}^{(k)}$ be the diagonal entries of $A_{k}$. Set $\underline{\hat{s}}=k^{-n / 2} \underline{s}$ so that $h_{\hat{s}}=h_{0}(k)$, where $h_{\hat{s}}$ is defined as in (4.2). Now let $\underline{\hat{s}}(t ; k)=$ $\left(e^{t \lambda_{0}} \hat{s}_{0}, e^{t \bar{\lambda}_{1}} \hat{s}_{1}, \ldots, e^{t \lambda_{N}} \hat{s}_{N}\right)$, and define

$$
h(t ; k)=h_{\underline{\hat{s}}(t ; k)}=h_{0} e^{-\phi(t ; k)}=h_{0}(k) e^{-(\phi(t ; k)-\phi(k))},
$$

so that $h(t ; k):(-\infty, 0] \rightarrow \mathcal{H}_{k}$ is a geodesic ray in $\mathcal{H}_{k}$ and $h(0 ; k)=h_{0}(k)$. In particular, we have

$$
\begin{aligned}
\phi(t ; k) & =\frac{1}{k} \log \left(k^{-n} \cdot \sum_{\alpha=0}^{N_{k}} e^{2 t \lambda_{\alpha}}\left|s_{\alpha}\right|_{h_{0}^{k}}^{2}\right) \\
& =\frac{1}{k} \log \left(k^{-n} \cdot \sum_{\alpha=0}^{N_{k}} e^{2 t \lambda_{\alpha}}\left|s_{\alpha}\right|_{h_{0}(k)^{k}}^{2}\right)+\phi(k) .
\end{aligned}
$$

Let

$$
f(k)=\frac{w(k)}{k d_{k}}-F_{0}=\frac{F(T)}{k}+O\left(\frac{1}{k^{2}}\right),
$$

where $w(k), d_{k}$ and $F_{0}$ are defined as in (2.2). In particular, $f(k)=O\left(\frac{1}{k}\right)$. 
Lemma 4.2. Let $k, l$ be positive integers with $k<l$. Then there exists $C_{k, l}>0$ with the following property:

$$
-C_{k, l}<[\phi(t ; l)+2 t \cdot f(l)]-[\phi(t ; k)+2 t \cdot f(k)]<C_{k, l} .
$$

Proof. If suffices to prove (4.12) in the case $k=1$. Then, replacing $l$ by $k$, we have

$$
\begin{array}{r}
{[\phi(t ; k)+2 t \cdot f(k)]-[\phi(t ; 1)+2 t \cdot f(1)]} \\
=\log \frac{\left(k^{-n} \cdot \sum_{\alpha=0}^{N_{k}} e^{2 t \eta_{\alpha}^{(k)}}\left|s_{\alpha}^{(k)}\right|_{h_{0}^{k}}^{2}\right)^{1 / k}}{\left(\sum_{\beta=0}^{N} e^{2 t \eta_{\beta}^{(1)}}\left|s_{\beta}\right|_{h_{0}}^{2}\right)},
\end{array}
$$

where $\eta_{0}^{(k)} \leq \eta_{1}^{(k)} \cdots \leq \eta_{N_{k}}^{(k)}$ are the eigenvalues of the diagonal matrix $B_{k}$, $N=N_{1}$ and $s_{\beta}=s_{\beta}^{(1)}$.

We now have

$$
\log \left(\sum_{\beta=0}^{N} e^{2 t \eta_{\beta}^{(1)}}\left|s_{\beta}\right|_{h_{0}}^{2}\right)=\frac{1}{k} \log \left(\sum_{\alpha=0}^{N_{k}} e^{2 t \eta_{\alpha}^{(k)}}\left|\tilde{s}_{\alpha}^{(k)}\right|_{h_{0}^{k}}^{2}\right)+O(1),
$$

where the $O(1)$ term is independent of $t$, and, for $\eta \in \mathbf{Z}$,

$$
\begin{gathered}
\left\{\tilde{s}_{\alpha}^{(k)}: \eta_{\alpha}^{(k)}=\eta\right\} \subset\left\{s_{0}^{p_{0}} \otimes \cdots \otimes s_{N}^{p_{N}} \in H^{0}\left(X, L^{k}\right): \sum_{\beta} p_{\beta}=k,\right. \\
\left.\quad \text { and } \sum_{\beta} p_{\beta} \eta_{\beta}^{(1)}=\eta\right\},
\end{gathered}
$$

is a maximally linearly independent subset. On the other hand, $\left(s_{0}^{(k)}, \ldots\right.$, $\left.s_{N_{k}}^{(k)}\right)$ and $\left(\tilde{s}_{0}^{(k)}, \ldots, \tilde{s}_{N_{k}}^{(k)}\right)$ are two bases of the same vector space which differ by a lower block triangular matrix. This proves Lemma 4.2.

4.3. The volume formula. Let $\phi_{t}:[a, b] \rightarrow \mathcal{H}_{\omega}$ be a smooth path and let $U_{a, b}=\left\{w \in \mathbf{C}^{\times}: e^{a} \leq|w| \leq e^{b}\right\}$. Let $M_{a, b}=X \times U_{a, b}$ and $\Omega_{0}$ be the $(1,1)$ form on $M_{a, b}$ defined by pulling back $\omega_{0}$. Define $\Phi(z, w): M_{a, b} \rightarrow \mathbf{R}$ by

$$
\Phi(z, w)=\phi_{t}(z) \quad \text { where } t=\log |w| \text {. }
$$

Let $\Omega_{\Phi}$ be the $(1,1)$ form on $M_{a, b}$ defined by $\Omega_{\Phi}=\Omega_{0}+\frac{\sqrt{-1}}{2} \partial \bar{\partial} \Phi$. Then

$$
\Omega_{\Phi}^{n+1}=\frac{1}{4}\left(\ddot{\phi}_{t}-\left|\partial \dot{\phi}_{t}\right|^{2}\right) \omega_{\phi_{t}}^{n} \wedge\left(\frac{\sqrt{-1}}{2} d w \wedge d \bar{w}\right) .
$$


In particular, we have the key observation of $[\mathbf{1 2}, \mathbf{1 9}, \mathbf{2 9}]$ :

$$
\Omega_{\Phi}^{n+1}=0 \Longleftrightarrow \ddot{\phi}_{t}-\left|\partial \dot{\phi}_{t}\right|_{\omega_{\phi_{t}}}^{2}=0
$$

We say that a function $\phi_{t}(x)$ on $[a, b] \times X$ is a weak geodesic if $\Phi$ is bounded, plurisubharmonic with respect to $\Omega_{0}$, and if $\Omega_{\Phi}^{n+1}=0$.

Finally, we obtain, using (3.5), the following useful volume formula [27]:

$$
(n+1) \int_{X \times U_{a, b}} \Omega_{\Phi}^{n+1}=\dot{E}_{\omega}(b)-\dot{E}_{\omega}(a),
$$

where $E_{\omega}(t)=E_{\omega}\left(\phi_{t}\right)$.

4.4. Volume estimates for the Monge-Ampère measure. We first need a few lemmas. Let $D^{\times}=\{w \in \mathbf{C}: 0<|w|<1\}$. We associate to $\phi(t ; k)$ the function $\Phi(k)$ on $X \times D^{\times}$as in (4.16):

$$
\Phi(k)(z, w)=\phi(t ; k)(z) \quad \text { where } t=\log |w|
$$

and we let $\Omega_{0}$ be the pullback of $\omega_{0}$ to $X \times D^{\times}$and we let $\Omega_{\Phi(k)}=\Omega_{0}+$ $\frac{\sqrt{-1}}{2} \partial \bar{\partial} \Phi(k)$.

Lemma 4.3. We have $\lim _{k \rightarrow \infty} \int_{X \times D^{\times}} \Omega_{\Phi(k)}^{n+1}=0$. In fact,

$$
\int_{X \times D^{\times}} \Omega_{\Phi(k)}^{n+1}=O\left(k^{-1}\right) .
$$

Proof. According to (4.19),

$$
\int_{X \times D^{\times}} \Omega_{\Phi(k)}^{n+1}=\int_{X} \dot{\phi}(0 ; k) \omega_{\phi(0 ; k)}^{n}-\lim _{t \rightarrow \infty} \int_{X} \dot{\phi}(t ; k) \omega_{\phi(t ; k)}^{n}
$$

Hence it suffices to show that each of the two terms in $(4.21)$ is $O\left(\frac{1}{k}\right)$.

Let $\psi(t ; k)=k[\phi(t ; k)-\phi(k)]+n \log k$. Then $\psi(t ; k)=\log \frac{\left|\sigma_{t} z\right|^{2}}{|z|^{2}}$, where $\sigma_{t}=e^{t A_{k}}$. Recall that $\omega_{0}+\frac{\sqrt{-1}}{2} \partial \bar{\partial} \phi(k)=\omega_{0}(k)$ and $\omega_{0}(k)=\frac{1}{k} \iota_{\underline{s}}^{*} \omega_{\mathrm{FS}}$. Thus

$$
\begin{aligned}
\omega_{\phi(t ; k)} & =\omega_{0}+\frac{\sqrt{-1}}{2} \partial \bar{\partial} \phi(t ; k)=\omega_{0}(k)+\frac{1}{k} \frac{\sqrt{-1}}{2} \partial \bar{\partial} \psi_{t} \\
& =\frac{1}{k} \iota_{s}^{*}\left(\omega_{\mathrm{FS}}+\frac{\sqrt{-1}}{2} \partial \bar{\partial} \psi_{t}\right) .
\end{aligned}
$$

Since we also have $\dot{\phi}(t ; k)=\frac{1}{k} \dot{\psi}(t ; k)$ we conclude that

$$
\begin{aligned}
(n+1) \int_{X} \dot{\phi}(t ; k) \omega_{\phi(t ; k)}^{n} & =(n+1) \cdot \frac{1}{k} \cdot \frac{1}{k^{n}} \cdot \int_{Z_{k}} \dot{\psi}_{t}\left(\omega_{\mathrm{FS}}+\frac{\sqrt{-1}}{2} \partial \bar{\partial} \psi_{t}\right)^{n} \\
& =\frac{1}{k} \cdot \frac{1}{k^{n}} \cdot \dot{E}_{\omega_{\mathrm{FS}}}\left(\psi_{t}\right) .
\end{aligned}
$$


Thus

$$
-\lim _{t \rightarrow-\infty} \int_{X} \dot{\phi}(t ; k) \omega_{\phi(t ; k)}^{n}=\frac{1}{(n+1)} \frac{1}{k} \frac{\mu\left(Z_{k}, A_{k}\right)}{k^{n}}
$$

Now, according to Lemma $3.3, \frac{\mu\left(Z_{k}, A_{k}\right)}{k^{n}}$ has a finite limit as $k$ tends to infinity. In fact, the limit is equal to $F(T)$, the Futaki invariant. Thus the second term in $(4.22)$ is $O\left(\frac{1}{k}\right)$.

In order to treat the first term, we require the following result from [16].

Lemma 4.4. Let $L \rightarrow X$ be an ample line bundle over a compact complex manifold $X$ and $h$ a metric on $L$ with positive curvature $\omega$. Let $\underline{s}$ be an orthonormal basis of $H^{0}\left(X, L^{k}\right)$ and let $\iota_{\hat{s}}: X \hookrightarrow \mathbf{P}^{N_{k}}$ be the associated Kodaira imbedding. Let $Z_{k}$ be the image of $\iota_{\underline{s}}$. Define $M_{\alpha \beta}^{(k)}=M_{\alpha \beta}\left(Z_{k}\right)$ as in (3.11). Then

$$
\begin{aligned}
M_{\alpha \beta}^{(k)} & =\int_{Z_{k}} \frac{z_{\alpha} \bar{z}_{\beta}}{|z|^{2}} \omega_{\mathrm{FS}}^{n}=\int_{X}\left(s_{\alpha}, s_{\beta}\right)_{h^{k}} \cdot\left(\frac{h(k)}{h}\right)^{k} \cdot \frac{\omega(k)^{n}}{\omega^{n}} \cdot \omega^{n} \\
& =\delta_{\alpha \beta}-k^{-1} \int_{X}\left(s_{\alpha}, s_{\beta}\right)_{h^{k}} \cdot s(\omega) \omega^{n}+O\left(k^{-2}\right) \\
& =\left(\frac{1}{n !} \int_{X} \omega^{n}\right) \frac{k^{n}}{N_{k}} \delta_{\alpha \beta}-k^{-1} \int\left(s_{\alpha}, s_{\beta}\right)_{h^{k}}[s(\omega)-\bar{s}] \omega^{n}+O\left(k^{-2}\right) \\
4.25) & =\left(\frac{1}{n !} \int_{X} \omega^{n}\right) \frac{k^{n}}{N_{k}} \delta_{\alpha \beta}-k^{-1} \int \frac{x_{\alpha} \bar{x}_{\beta}}{|x|^{2}}[s(\omega)-\hat{s}] \omega_{\mathrm{FS}}^{n}+O\left(k^{-2}\right),
\end{aligned}
$$

where $\hat{s} \in \mathbf{R}$ is defined by: $\int_{X}[s(\omega)-\hat{s}] \omega^{n}=0$. The proof of Lemma 4.4 follows from (4.7).

We return to the proof of Lemma 4.3: Applying (4.25) to (3.10) we obtain:

$$
\begin{aligned}
\frac{1}{n+1} \int_{X} \dot{\phi}(0 ; k) \omega_{\phi(0 ; k)}^{n}= & \frac{1}{k} \cdot \frac{1}{k^{n}} \cdot \dot{E}_{\omega_{\mathrm{FS}}}\left(\psi_{t}\right)=\frac{1}{k^{n}} \frac{2}{k} \operatorname{tr}\left(A_{k} M^{(k)}\right) \\
= & -\frac{1}{k^{n}} \frac{2}{k} \int \sum_{\alpha}\left(s_{\alpha}, s_{\alpha}\right)_{h^{k}} \frac{\lambda_{\alpha}^{(k)}}{k}[s(\omega)-\hat{s}] \omega^{n} \\
& +O\left(k^{-2}\right) \frac{1}{k^{n}} \frac{1}{k} \sum_{\alpha}\left|\lambda_{\alpha}\right|
\end{aligned}
$$

where in the first equality we use the fact that $A_{k}=A_{k}^{*}$ and in the last equality we have made use of the fact that $A_{k}$ is traceless.

If we apply Lemma 3.1 to equation (4.26) we obtain

$$
\left|\int_{X} \dot{\phi}(0 ; k) \omega_{\phi(0 ; k)}^{n}\right| \leq C^{\prime} \frac{1}{k^{n}} \frac{1}{k} \sum_{\alpha} \int\left|s_{\alpha}\right|_{h^{k}}^{2} \omega^{n}+O\left(k^{-2}\right) \frac{1}{k^{n}} \frac{1}{k} C k N_{k},
$$


where $C^{\prime}=C \sup _{X}|s(\omega)-\hat{s}|$. The first term equals $C^{\prime} \frac{1}{k} \frac{N_{k}+1}{k^{n}}$. Since $\frac{N_{k}+1}{k^{n}}$ is bounded as a function of $k$, the first term is $O\left(k^{-1}\right)$. Similarly, the second term is $O\left(k^{-2}\right)$.

4.5. The Monge-Ampère equation on a punctured disk. We now complete the proof of Theorem 1.1. As in the proof of [27, Theorem 3], we can choose a sequence of positive real numbers $c_{k} \searrow 0$ in such a way that

$$
\phi(0 ; k)+c_{k}>\phi(0 ; k+1)+c_{k+1} .
$$

Indeed, by the Tian-Yau-Zelditch theorem, $\sup _{X}|\phi(0 ; k)-\phi| \leq C k^{-2}$, so that the sequence $c_{k}=2 C \sum_{j \geq k} j^{-2}$ is such a choice. Choose also $\epsilon_{k}=$ $k^{-1 / 2}$ and make the replacement

$$
\phi(t ; k) \longrightarrow \phi(t ; k)+c_{k}-\epsilon_{k} t .
$$

Then it is still true that $\phi(0 ; k) \rightarrow \phi$, and that $\int \Omega_{\Phi(k)}^{n+1}=O\left(\frac{1}{k}\right)$. Moreover, the value of $\phi_{t}$, as defined in (1.1) does not change under this replacement.

Next, we show that $\phi_{t}$ is continuous at $t=0$ and has the desired initial value. As in [27], the essential ingredient is a uniform bound for $|Y \phi(t ; k)|$ near the boundary $S^{1} \times X$, where $Y=\partial_{t}$. In fact, differentiating the expression for $\phi(t ; k)$ gives

$$
\begin{aligned}
|\dot{\phi}(t ; k)| & \leq \frac{2}{k} \frac{\sum_{\alpha=0}^{N}\left|\lambda_{\alpha}\right| e^{2 t \lambda_{\alpha}}\left|s_{\alpha}\right|^{2}}{\sum_{\alpha=0}^{N} e^{2 \lambda_{\alpha}}\left|s_{\alpha}\right|^{2}}+\epsilon_{k} \\
& \leq \frac{2}{k} \sup _{\alpha}\left|\lambda_{\alpha}\right|+\epsilon_{k} \leq C,
\end{aligned}
$$

where in the last step, we made use of the bound $\left\|A_{k}\right\|_{\text {op }} \leq C k$ provided by Lemma 3.1. On the boundary $X \times S^{1}$, the monotonicity of $\phi(t ; k)$ guarantees that, for any pair $k, l$ with $k<l$,

$$
\phi(t ; k)-\phi(t ; l)>\phi(t ; k)-\phi(t ; k+1)>\delta_{k} \quad \text { on } X \times S^{1},
$$

where $\delta_{k}$ is a strictly positive constant independent of $l$. Since $|\dot{\phi}(t ; m)|$ is uniformly bounded in $m$, it follows that $\phi(t ; k)-\phi(t ; l)>\frac{1}{2} \delta_{k}$ in a neighborhood $U_{k}$ of $X \times S^{1}$ independent of $l$. Thus, we have for any $k$,

$$
\left[\sup _{l \geq k} \phi(t ; l)\right]^{*}=\phi(t ; k)
$$

in an open neighborhood $U_{k}$ of $X \times S^{1}$. Extend now the original potential $\phi$ on $X$ as a function in a neighborhood of $X \times S^{1}$, by making it constant along the flow lines of $Y$. For any $\epsilon>0$, choose $k$ large enough so that $\sup _{X}|\phi(0 ; k)-\phi|<\epsilon$. Then the above estimate for $\dot{\phi}(t ; k)$ shows that we have

$$
\sup _{U}|\phi(t ; k)-\phi|<2 \epsilon
$$


for some neighborhood $U$ of $X \times S^{1}$ in $X \times D^{\times}$, independent of $k$. This implies that $\phi_{t}=\lim _{k \rightarrow \infty}\left[\sup _{l \geq k} \phi(t ; k)\right]^{*}$ is continuous at $X \times S^{1}$, and that $\phi_{t}=\phi$ at $t=0$.

On the other hand, for fixed $l \geq j>k>0$, Lemma 4.2 implies that

$$
\begin{aligned}
\phi(t ; j)-\phi(t ; k) & \leq C_{k, j}+c_{j}-c_{k}+2 t f(k)-2 t f(j)-\left(\epsilon_{k}-\epsilon_{j}\right)|t| \\
& \leq\left[\sup _{k<m \leq l} C_{k, m}\right]+1-\frac{1}{2}\left(\epsilon_{k}-\epsilon_{k+1}\right)|t|,
\end{aligned}
$$

for $k$ sufficiently large. Thus, we can make sure that

$$
\phi(t ; k)>1+\phi(t ; j)
$$

for all $j$ such that $k<j \leq l$ and all $t$ such that

$$
|t|>2 \frac{2+\sup _{k<m \leq l} C_{k, m}}{\epsilon_{k}-\epsilon_{k+1}} \equiv-\log r_{k, l} .
$$

Clearly, we have $r_{k, l+1}<r_{k, l}$ and, by choosing $C_{k, l}$ in Lemma 4.2 large enough, we can make sure that

$$
\lim _{l \rightarrow \infty} r_{k, l}=\lim _{l \rightarrow \infty} r_{l, l+1}=0
$$

for each $k>0$. Thus, if we set

$$
\phi(t ; k, l)=\sup _{k \leq j \leq l}[\phi(t ; j)],
$$

and let $\Omega_{k, l}$ be the $(1,1)$ form on $X \times D^{\times}$corresponding to $\phi(t ; k, l)$ via (4.16), we have, for $l>k$,

$$
\begin{aligned}
\int_{X \times D_{r_{k, k+1}}} \Omega_{k, l}^{n+1} & \leq \int_{X \times D_{r_{k, l}}} \Omega_{k, l}^{n+1}=\int_{X \times D_{r_{k, l}}} \Omega_{\Phi(k)}^{n+1} \\
& \leq \int_{X \times D^{\times}} \Omega_{\Phi(k)}^{n+1} \leq \frac{C}{k}
\end{aligned}
$$

where $C$ is independent of $k, l$ and

$$
D_{r}=\{w \in \mathbf{C}: 1>|w|>r\} .
$$

In the middle equality above, we made use of the fact that the volume integrals depend only on the values of the currents in a neighborhood of the boundary of $X \times D_{r_{k, l}}$ (see, [27, Lemma 2]).

Now $\phi(t ; k, l)$ is an increasing sequence in the index $l$ which converges pointwise, almost everywhere, to $\xi(t ; k)=\sup _{k \leq j}[\phi(t ; j)]^{*}$. Let $\Xi_{k}$ be the $(1,1)$ form on $D^{\times} \times M$ corresponding to $\xi(t ; k)$. Then, by the BedfordTaylor monotonicity theorem [3] applied to the increasing sequence $\phi(t ; k, l)$ (see also Blocki [6] and Cegrell [8]), we have

$$
\int_{X \times D_{r_{k, k+1}}} \Xi_{k}^{n+1}=\lim _{l \rightarrow \infty} \int_{X \times D_{r_{k, k+1}}} \Omega_{k, l}^{n+1} \leq \frac{C}{k} .
$$


Moreover, if $l \geq k$,

$$
\int_{X \times D_{r_{k, k+1}}} \Xi_{l}^{n+1} \leq \int_{X \times D_{r_{l, l+1}}} \Xi_{l}^{n+1} \leq \frac{C}{l} .
$$

Finally, since $\xi(t ; l)$ is monotonically decreasing to $\phi(t)$ (by definition of $\phi(t)$ ) we have, using the Bedford-Taylor monotonicity theorem again (but this time for decreasing sequences):

$$
\int_{X \times D_{r_{k, k+1}}} \Omega_{\Phi}^{n+1}=\lim _{l \rightarrow \infty} \int_{X \times D_{r_{k, k+1}}} \Xi_{l}^{n+1}=0 .
$$

Since this is true for all $k$, we obtain

$$
\int_{X \times D^{\times}} \Omega_{\Phi}^{n+1}=0
$$

Thus $\Omega_{\Phi}^{n+1}=0$, and this proves the theorem.

\section{Proof of Theorem 1.2}

In this section, we show that if the expression $N_{2}(T)^{2}$ defined below by (5.3) is strictly positive, then $\phi_{t}$ is a non-trivial geodesic, i.e., $\phi_{t}$ is not a constant function of $t$.

Let $N_{1}+1=\operatorname{dim}(X, L)$ and set $N=N_{1}$. Let $\lambda_{0} \geq \lambda_{1} \geq \cdots \geq \lambda_{N}$ be the diagonal entries of $A_{1}$ and let $N_{1}+1=\operatorname{dim} H^{0}(X, L)$. By Lemma 2.1 we may assume that $T$ is imbedded in $\mathbf{P}^{N}$ and that the action $\rho(\tau)$ is given by the diagonal matrix whose diagonal entries are given by $\tau^{\lambda_{0}}, \ldots, \tau^{\lambda_{N}}$. As usual, we denote by $X_{0} \subseteq \mathbf{P}^{N}$ the central fiber of $T$.

Define $h: \mathbf{P}^{N} \rightarrow \mathbf{R}$ by

$$
h(z)=\frac{\sum_{\alpha=0}^{N} \lambda_{\alpha}\left|z_{\alpha}\right|^{2}}{\sum_{\alpha=0}^{N}\left|z_{\alpha}\right|^{2}} .
$$

We next recall the formula in Donaldson [16]:

$$
\operatorname{Tr}\left(A_{k}^{2}\right)=N_{2}(T)^{2} \cdot k^{n+2}+O\left(k^{n+1}\right),
$$

where the coefficient $N_{2}(T)$ is given by

$$
N_{2}(T)^{2}=\int_{X_{0}}(h-\hat{h})^{2} \omega_{\mathrm{FS}}^{n},
$$

and $\hat{h}$ is determined by $\int_{X_{0}}(h-\hat{h}) \omega_{\mathrm{FS}}^{n}=0$. The test configuration $T$ is trivial if and only if $N_{2}(T)=0$.

Now let $\lambda=\lambda_{N}$ so that $\lambda \leq \lambda_{\alpha}$ for all $\alpha$. Denote by $\lambda_{0}^{(k)} \geq \lambda_{1}^{(k)} \geq$ $\cdots \geq \lambda_{N_{k}}^{(k)}$ the eigenvalues of the endomorphism $A_{k}$ (for convenience, the 
eigenvalues are ordered here in the opposite order than previously). Set $\lambda^{(k)}=\lambda_{N_{k}}^{(k)}$. Then $\lambda^{(k)}=k \lambda-\frac{\operatorname{Tr} B_{k}}{N_{k}}$, and $\lambda^{(k)} k^{-1}$ has a limit. Set

$$
\Lambda=\lim _{k \rightarrow \infty} \frac{\lambda^{(k)}}{k} \text {. }
$$

If $N_{2}(T)>0$ then (5.3) implies that the average absolute eigenvalue $\left|\lambda_{j}^{(k)}\right|$ has size at least $k$. On the other hand, since $\operatorname{Tr} A_{k}=0$, one easily sees that $\left|\lambda^{(k)}\right|$ has size at least $k$ and thus $\Lambda>0$.

Next, recall that

$$
\phi(t ; k)=\frac{1}{k} \log \left(k^{-n} \cdot \sum_{\alpha=0}^{N_{k}} e^{2 t \lambda_{\alpha}^{(k)}}\left|s_{\alpha}^{(k)}\right|_{h_{0}^{k}}^{2}\right) .
$$

Observe that

so

$$
\int \sum_{\alpha=0}^{N_{k}} e^{2 t \lambda_{\alpha}}\left|s_{\alpha}\right|_{h_{0}^{k}}^{2} \omega_{0}^{n} \geq e^{2\left|t \lambda^{(k)}\right|}
$$

$$
\begin{aligned}
2|t| \frac{\left|\lambda^{(k)}\right|}{k}+O\left(\frac{1}{k^{2}}\right) \geq & \sup _{X_{t}} \phi(t ; k) \geq 2|t| \frac{\left|\lambda^{(k)}\right|}{k}-n \frac{\log k}{k} \\
& -\frac{1}{k} \log \left(\int_{X} \omega_{0}^{n}\right)
\end{aligned}
$$

so, letting $k \rightarrow \infty$,

$$
\sup _{X_{t}} \phi_{t}=2|t| \cdot|\Lambda|
$$

Since $|\Lambda|>0$, this already shows that $\phi_{t}$ is non-trivial if $N_{2}(T)>0$. This establishes Theorem 2.

Under the additional technical assumption (which we expect can be removed) that for $k_{0}$ large enough, $\left[\sup _{k \geq k_{0}} \phi(t ; k)\right]^{*}=\sup _{k \geq k_{0}} \phi(t ; k)$ for $|t|>t_{k_{0}} \gg 1$, then the geodesic $\phi_{t}$ can be shown to be non-trivial in the stronger sense that it defines a non-trivial ray in $\mathcal{H} / \mathbf{R}$.

To show strong non-triviality, we observe that $N_{2}(T)>0$ implies (and is in fact, equivalent to) the following. There exist $p \in X$ such that $s_{\alpha}(p)=0$ for all $\alpha$ such that $\lambda_{\alpha}=\lambda$. Fix such a $p$. Let $\gamma=\inf \left\{\lambda_{\alpha}: \lambda_{\alpha}>\lambda\right\}$ and $\gamma^{(k)}=\inf \left\{\lambda_{\alpha}^{(k)}: \lambda_{\alpha}^{(k)}>\lambda^{(k)}\right\}$. Note that $|\gamma|<|\lambda|$. Again, $\gamma^{(k)}=k \gamma-\frac{\operatorname{Tr} B_{k}}{N_{k}}$, and $\frac{\gamma^{(k)}}{k}$ has a limit as $k \rightarrow \infty$,

$$
\Gamma=\lim _{k \rightarrow \infty} \frac{\gamma^{(k)}}{k}
$$

satisfying $|\Gamma|<|\Lambda|$. Now, at the point $p$, we have for all $k$,

$$
\phi(t ; k)(p) \leq 2|t| \frac{\left|\gamma^{(k)}\right|}{k}+O\left(\frac{1}{k}\right) .
$$


Fix $\epsilon>0$ so small that $|\Gamma|+2 \epsilon<|\Lambda|$. Then there exists $k_{0}$ so that

$$
\phi(t ; k)(p) \leq 2(|\Gamma|+\epsilon)|t|
$$

for all $|t|>1$ and all $k \geq k_{0}$. We have then, for $|t|$ sufficiently large,

$$
\begin{aligned}
\phi_{t}(p) & \leq\left[\sup _{k \geq k_{0}} \phi(t ; k)\right]^{*}(p) \\
& =\sup _{k \geq k_{0}} \phi(t ; k)(p) \leq 2(|\Gamma|+\epsilon)|t| .
\end{aligned}
$$

In view of (5.6), this shows $\lim _{t \rightarrow-\infty} \operatorname{osc}_{X_{t}} \phi_{t}=\infty$ where $\operatorname{osc}_{X_{t}} \phi_{t}=$ $\sup _{X_{t}} \phi_{t}-\inf _{X_{t}} \phi_{t}$. Thus $\phi_{t}$ is strongly non-trivial.

\section{Proof of Theorem 1.3}

The formula in Lemma 8.8 of Tian [31] implies that

$$
\lim _{t \rightarrow-\infty} \dot{\nu}_{k}=F_{\mathrm{CM}}(T)
$$

where $F_{\mathrm{CM}}(T)$ is the CM-Futaki invariant (see $[\mathbf{3 1}]$ for the precise definition).

On the other hand, the recent work of Paul-Tian $[\mathbf{2 2}]$ shows that $F_{\mathrm{CM}}(T)=F(T)$ under the hypothesis of Theorem 1.3.

\section{References}

[1] C. Arezzo and G. Tian, Infinite geodesic rays in the space of Kähler potentials, Ann. Sci. Norm. Sup. Pisa Sci. (5) 2 (2003), 617-630, arXiv:math.DG/0210389.

[2] E. Bedford, and B.A. Taylor, The Dirichlet problem for a complex Monge-Ampère equation, Invent. Math. 37 (1976), 1-44.

[3] E. Bedford and B.A. Taylor, A new capacity for plurisubharmonic functions, Acta Math. 149 (1982), 1-40.

[4] B. Berndtsson, Curvature of vector bundles associated to holomorphic fibrations, preprint, CV/0511225.

[5] B. Berndtsson, Positivity properties of direct image bundles, preprint, CV/0608385.

[6] Z. Blocki, The complex Monge-Ampère operator and pluripotential theory, http://gamma.im.uj.edu.pl/ blocki/publ/ln/index.html.

[7] D. Catlin, The Bergman kernel and a theorem of Tian, in 'Analysis and geometry in several complex variables (Katata, 1997)', Trends in Mathematics, Birkhäuser Boston, Boston, MA, 1999, 1-23.

[8] U. Cegrell, Capacities in complex analysis, Aspects of Mathematics, E14, Vieweg, 1988.

[9] X.X. Chen, The space of Kähler metrics, J. Diff. Geom. 56 (2000), 189-234. 
[10] X.X. Chen, Space of Kähler metrics III - On the lower bound of the Calabi energy and geodesic distance, arXiv:math.DG/0606228.

[11] X.X. Chen, and G. Tian, Geometry of Kähler metrics and foliations by discs, arXiv:math.DG/0409433.

[12] S.K. Donaldson, Symmetric spaces, Kähler geometry, and Hamiltonian dynamics, Amer. Math. Soc. Transl. 196 (1999), 13-33.

[13] S.K. Donaldson, Scalar curvature and projective imbeddings I, J. Diff. Geom. 59 (2001), 479-522.

[14] S.K. Donaldson, Scalar curvature and stability of toric varieties, J. Diff. Geom. 62 (2002), 289-349.

[15] S.K. Donaldson, Scalar curvature and projective imbeddings II, arXiv:math.DG/0407534.

[16] S.K. Donaldson, Lower bounds on the Calabi functional, J. Diff. Geom. 70 (2005), 453-472.

[17] S. Kolodziej, The complex Monge-Ampère equation, Acta Math. 180(1) (1998), 69-117.

[18] Z. Lu, On the lower order terms of the asymptotic expansion of TianYau-Zelditch, Amer. J. Math. 122 (2000), 235-273.

[19] T. Mabuchi, Some symplectic geometry on compact Kähler manifolds, Osaka J. Math. 24 (1987), 227-252.

[20] D. Mumford, Stability of projective varieties, Enseignement Math. 23 (1977), 39-110.

[21] S. Paul, Geometric analysis of Chow Mumford stability, Adv. Math. 182 (2004), 333-356.

[22] S. Paul, and G. Tian, CM stability and the generalized Futaki invariant, arXiv:math:AG/0605278.

[23] S. Paul, and G. Tian, CM stability and the generalized Futaki invariant II, arXiv:math.DG/0606505

[24] D.H. Phong, and J. Sturm, Stability, energy functionals, and Kähler-Einstein metrics, Comm. Anal. Geom. 11 (2003), 563-597, arXiv:math.DG/0203254.

[25] D.H. Phong, and J. Sturm, Scalar curvature, moment maps, and the Deligne pairing, Amer. J. Math. 126 (2004), 693-712, arXiv:math.DG/0209098.

[26] D.H. Phong, and J. Sturm, The Futaki invariant and the Mabuchi energy of a complete intersection, Comm. Anal. Geom. 12 (2004), 321-343, arXiv:math.DG/0312529.

[27] D.H. Phong, and J. Sturm, The Monge-Ampère operator and geodesics in the space of Kähler potentials, Invent. Math. 166 (2006), 125-149, arXiv:math.DG/0504157.

[28] J. Ross, and R. Thomas, A study of the Hilbert-Mumford criterion for the stability of projective varieties, arXiv:math.AG/0412519. 
[29] S. Semmes, Complex Monge-Ampère and symplectic manifolds, Amer. J. Math. 114 (1992), 495-550.

[30] G. Tian, On a set of polarized Kähler metrics on algebraic manifolds, J. Diff. Geom. 32 (1990), 99-130.

[31] G. Tian, Kähler-Einstein metrics with positive scalar curvature, Invent. Math. 130 (1997), 1-37.

[32] S.T. Yau, On the Ricci curvature of a compact Kähler manifold and the complex Monge-Ampère equation I, Comm. Pure Appl. Math. 31 (1978), 339-411.

[33] S.T. Yau, Nonlinear analysis in geometry, Enseign. Math. (2) 33 (1-2) (1987), 109-158.

[34] S.T. Yau, Open problems in geometry, Proceedings of Symposia in Pure Mathematics, 54, AMS Providence, RI, 1993, 1-28.

[35] S. Zelditch, The Szegö kernel and a theorem of Tian, Int. Math. Res. Not. 6 (1998), 317-331.

[36] S. Zhang, Heights and reductions of semi-stable varieties, Compos. Math. 104 (1996), 77-105.

Department of Mathematics

COLUMBIA UNIVERSITY

NEW YORK NY 10027

E-mail address: phong@math.columbia.edu

Department of Mathematics

Rutgers UNIVERSITY

NEWARK NJ 07102

E-mail address: sturm@rutgers.edu

Received 08/09/2006, accepted 08/07/2007

Research supported in part by National Science Foundation grants DMS-02-45371 and DMS-05-14003. 
